\title{
Discontinuities without discontinuity: The Weakly-enforced Slip Method
}

\author{
G.J. van Zwieten ${ }^{1,3 *}$ E.H. van Brummelen ${ }^{1,2}$, K.G. van der Zee ${ }^{1}$, \\ M.A. Gutiérrez ${ }^{3}$, R.F. Hanssen ${ }^{4}$ \\ 1 Eindhoven University of Technology, \\ Department of Mechanical Engineering, \\ P.O. Box 513, 5600 MB Eindhoven, The Netherlands \\ 2 Eindhoven University of Technology, \\ Department of Mathematics \& Computer Science, \\ P.O. Box 513, 5600 MB Eindhoven, The Netherlands \\ 3 Delft University of Technology, \\ Department of Mechanical, Maritime and Materials Engineering, \\ P.O. Box 5058, 2600 GB Delft, The Netherlands \\ 4 Delft University of Technology, \\ Department of Civil Engineering and Geosciences, \\ P.O. Box 5048, 2600 GA Delft, The Netherlands
}

March 8, 2021

\begin{abstract}
Tectonic faults are commonly modelled as Volterra or Somigliana dislocations in an elastic medium. Various solution methods exist for this problem. However, the methods used in practice are often limiting, motivated by reasons of computational efficiency rather than geophysical accuracy. A typical geophysical application involves inverse problems for which many different fault configurations need to be examined, each adding to the computational load. In practice, this precludes conventional finite-element methods, which suffer a large computational overhead on account of geometric changes. This paper presents a new non-conforming finite-element method based on weak imposition of the displacement discontinuity. The weak imposition of the discontinuity enables the application of approximation spaces that are independent of the dislocation geometry, thus enabling optimal reuse of computational components. Such reuse of computational components renders finite-element modeling a viable option for inverse problems in geophysical applications. A detailed analysis of the approximation properties of the new formulation is provided. The analysis is supported by numerical experiments in $2 \mathrm{D}$ and $3 \mathrm{D}$.
\end{abstract}

Keywords: Volterra dislocation, Finite Element Method, weak imposition, linear elasticity, tectonophysics.

*email: g.j.v.zwieten@tue.nl 


\section{Introduction}

The world is perpetually reminded of the fact that seismic hazard is still beyond reach of prediction - as it was most recently by the disaster that struck Japan. The difficulty is not just to predict the exact moment of failure, which, as argued by some [9], might never reach a level of practicality. It is also the nature of the risk, and the extent to which stress is accumulating, that turns out to be surprisingly difficult to constrain. The 2011 Tohoku-Oki earthquake demonstrated a great lack of understanding of ongoing tectonics [12. Arguably, a better understanding could have reduced the secondary effects if such information had led to more apt measures and regulations.

The main reason for this poor state of information can be traced to the absence of direct measurements. The primary quantities of interest, being the magnitude and orientation of the stress tensor in the earth's crust, can be obtained only through tedious, expensive, point-wise measurements. A viable broad scale method to directly measure the global stress field does not exist. For this reason information is obtained mostly from secondary observables, earthquakes themselves being an important source. Earthquakes represent significant, near instantaneous changes in the global stress field. By accurately determining the location of the segment of the fault that collapsed, one can progressively update the stress field and evolve it in time. This way the tectonic evolution is monitored, and hazardous areas can be identified as regions where stress accumulates. For successful tracking of stress development, however, it is essential to understand the tectonic mechanism behind every earthquake. This includes the location and geometry of the section of the fault that collapsed, and the direction and magnitude of fault slip. It is increasingly popular to base such analyses on local co-seismic surface displacements. This type of information has become available since the nineties with the advent of space borne interferometric SAR measurements of the earth's surface, and with the widespread availability of GPS measurements [19. Analysis of this data has in recent years seen rapid adoption and is now routinely performed for all major earthquakes.

A mechanical model is required to connect observations to physics. Most commonly (if not exclusively) used is an elastic dislocation model, based on the assumption that on short time scales, nonlinear (plastic) effects are negligible. The model embeds a displacement discontinuity of given location and magnitude in an elastic medium, causing the entire medium to deform under the locked-in stress. Many different solution methods have been developed for this particular problem, based on analytical solutions or numerical approximations; see for instance [21] for an overview of the most prominent methods. However, methods founded on analytical solutions generally dictate severe model simplifications, such as elastic homogeneity or generic geometries, which restricts their validity. The computational complexity of methods based on numerical approximation, on the other hand, is typically prohibitive in practical applications. Because in practice the surface displacements are given, and the dislocation parameters are the unknowns, the computational setting is always that of an inverse problem. A typical inversion requires several thousands of evaluations of the forward model, and therefore computational efficiency is a key requirement. Moreover, the forward problems in the inversion process are essentially identical, except for the fault geometry. Reuse of computational components, such as approximate factors of the system matrix, is imperative for efficiency of the inversion. Current numerical methods for seismic problems do not offer such reuse options.

Finite-element methods provide a class of numerical techniques that are particularly versatile in terms of modeling capabilities in geophysics. Finite-element methods allow for elastic heterogeneity, anisotropy, and topography; all things that can not well be accounted for with currently used analytical and semi-analytical methods. In geophysical practice, finite-element methods are however 
often rejected for reasons of computational cost. The high computational cost can be retraced to the condition that the geometry of the fault coincides with element edges, which is a requirement engendered by the strong enforcement of the dislocation; see [14. Consequently, the mesh geometry depends on the fault, which in turn implies that mesh-dependent components such as the stiffness matrix and approximate factorizations of that matrix cannot be reused for different fault geometries and must be recomputed whenever the geometry of the fault changes. The recomputation of these components in each step of a nonlinear inversion process leads to a prohibitive overall computational complexity.

To overcome the complications of standard finite-element techniques in nonlinear inversion processes in tectonophysics, this paper introduces the Weakly-enforced Slip Method (WSM), a new numerical method in which displacement discontinuities are weakly imposed. The WSM formulation is similar to Nitsche's variational principle for enforcing Dirichlet boundary conditions [15. The weak imposition of the discontinuity in WSM decouples the finite element mesh from the geometry of the fault, which renders the stiffness matrix and derived objects such as approximate factors independent of the fault and enables reuse of these objects. Therefore, even though the computational work required for a single realisation of the fault geometry is comparable to that of standard FEM, reuse of components makes WSM significantly more efficient when many different fault geometries are considered. This makes finite-element computations based on WSM a viable option for nonlinear inverse problems.

A characteristic feature of WSM is that it employs standard continuous finite-element approximation spaces, as opposed to the conventional FEM split-node approach [14] which introduces actual discontinuities in the approximation space. Instead, WSM approximations feature a 'smeared out' jump with sharply localized gradients. We will establish that the error in the WSM approximation converges only as $O\left(h^{1 / 2}\right)$ in the $\boldsymbol{L}^{2}$-norm as the mesh width $h$ tends to zero and that the error diverges as $O\left(h^{-1 / 2}\right)$ in the energy norm. In addition, however, we will show that the WSM approximation displays optimal local convergence in the energy norm, i.e., optimal convergence rates are obtained on any subdomain excluding a neighborhood of the dislocation. The numerical experiments convey that WSM also displays optimal local convergence in the $\boldsymbol{L}^{2}$-norm.

The remainder of this manuscript is organized as follows. Section 2 presents strong and weak formulations of Volterra's dislocation problem, and derives the corresponding lift-based finite-element formulation. Section 3 introduces the Weakly-enforced Slip Method based on two formal derivations, viz., by collapsing the support of the lift onto the fault and by application of Nitsche's variational principle. In Section 4, we examine the approximation properties of the WSM formulation. Section 5 verifies and illustrates the approximation properties on the basis of numerical experiments for several $2 \mathrm{D}$ and $3 \mathrm{D}$ test cases. In addition, to illustrate the generality of WSM, in the numerical experiments we consider several test cases that violate the conditions underlying the error estimates in Section 4, such as discontinuous slip distributions and rupturing dislocations. Section 6 presents concluding remarks.

\section{Problem formulation}

In this section we define Volterra's dislocation problem. We will postulate the strong formulation in 2.1. followed by a derivation of the weak formulation in 2.2 . The latter will serve as a basis for the derivation of the Finite Element approximations, for which we lay foundations in Section 2.3 . 


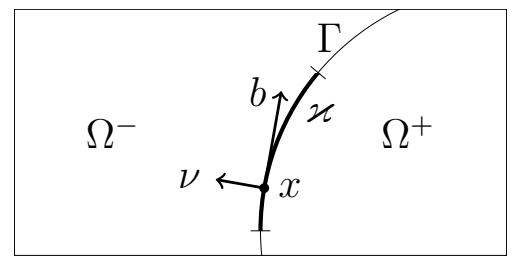

Figure 1: Definition of the domain $\Omega$, normal vector $\nu$, slip vector $b$, fault $\Gamma$ and dislocation $\varkappa$.

\subsection{The strong form}

We start by defining the geometric setup. We consider an open bounded domain $\Omega \subset \mathbb{R}^{N}(N=2,3)$ with Lipschitz boundary $\partial \Omega$. An $(N-1)$-dimensional Lipschitz manifold $\Gamma$, referred as the fault, divides the domain in two disjoint open subdomains $\Omega^{+}$and $\Omega^{-}$, such that $\Omega=\operatorname{int}\left(\overline{\Omega^{+}} \cup \overline{\Omega^{-}}\right)$. We equip $\Gamma$ with a unit normal vector $\nu: \Gamma \rightarrow \mathbb{R}^{N}$ directed into the subdomain $\Omega^{-}$. The fault supports a slip distribution $b: \Gamma \rightarrow \mathbb{R}^{N}$, corresponding to a dislocation. The fault is referred to as a non-rupturing fault if the dislocation $\varkappa=\operatorname{supp}(b)$ is compact in $\Gamma$, and a rupturing fault otherwise. Let us note that in tectonics, rupturing faults correspond to intersections of the slip plane with the surface of the earth. Figure 1 illustrates this setup for $N=2$. It is to be noted that $b$ need not be tangential to the fault. If $b$ has a non-vanishing normal component then the fault is opening, such as may be caused by an intruding material.

The displacement field generated by the dislocation is represented by $u: \Omega \backslash \Gamma \rightarrow \mathbb{R}^{N}$. For convenience, we restrict our considerations to linear elasticity. We denote by the map $u \mapsto \epsilon(u)$ the strain tensor corresponding to the displacement field $u$, according to

$$
\epsilon(u):=\frac{1}{2}\left[\nabla u+(\nabla u)^{T}\right]
$$

under the assumption that $u$ is differentiable on $\Omega \backslash \Gamma$. The constitutive behavior corresponds to Hooke's law:

$$
\sigma(u):=A: \epsilon(u),
$$

i.e., $\sigma_{i j}(u)=A_{i j k l} \epsilon_{k l}(u)$, where we adhere to the convention on summation on repeated indices. The tensors $\sigma$ and $A$ are referred to as the stress tensor and the elasticity tensor, respectively. The elasticity tensor is subject to the usual symmetries $A_{i j k l}=A_{i j l k}=A_{k l i j}$. Moreover, we assume that it is bounded and satisfies a strong positivity condition, i.e., there exist positive constants $\bar{c}_{A}>0$ and $\underline{c}_{A}>0$ such that:

$$
\underline{c}_{A} e_{i j} e_{i j} \leq A_{i j k l} e_{i j} e_{k l} \leq \bar{c}_{A} e_{i j} e_{i j}
$$

for all tensors $e$. The elasticity tensor is in principle allowed to vary over the domain $\Omega$, subject to the above conditions. Auxiliary smoothness conditions on $A$ will be introduced later.

To facilitate the formulation, we denote by $\sigma_{n}(u):=\sigma(u) \cdot n$ the traction on a boundary corresponding to the displacement field $u$. Moreover, we introduce the jump operator $[[\cdot]]$ and average operator $\{\cdot\}$ according to:

$$
\begin{aligned}
& {\left[[v]: \Gamma \ni x \mapsto v^{+}(x)-v^{-}(x),\right.} \\
& \{v\}: \Gamma \ni x \mapsto \frac{1}{2}\left[v^{+}(x)+v^{-}(x)\right],
\end{aligned}
$$


where $v^{+}$and $v^{-}$represent the traces of $v$ from within $\Omega^{+}$and $\Omega^{-}$, respectively. Given a partition of the boundary $\partial \Omega$ into $\mathcal{D} \neq \emptyset$ and $\mathcal{N}$ such that $\mathcal{D} \cap \mathcal{N}=\emptyset$, we define the Volterra dislocation problem as follows:

Strong formulation: given a body force $f: \Omega \rightarrow \mathbb{R}^{N}$, a displacement $g: \mathcal{D} \rightarrow \mathbb{R}^{N}$ and a traction $h: \mathcal{N} \rightarrow \mathbb{R}^{N}$, and slip $b: \Gamma \rightarrow \mathbb{R}^{N}$, find displacement field $u: \Omega \backslash \Gamma \rightarrow \mathbb{R}^{N}$ such that

$$
\begin{aligned}
-\operatorname{div} \sigma(u) & =f \text { in } \Omega \backslash \Gamma \\
{[u]] } & =b \text { on } \Gamma \\
{\left.\left[\sigma_{\nu}(u)\right]\right] } & =0 \text { on } \Gamma \\
u & =g \text { on } \mathcal{D} \\
\sigma_{n}(u) & =h \text { on } \mathcal{N}
\end{aligned}
$$

Equation $5 \mathrm{aa}$ is the usual equilibrium condition, which applies everywhere in $\Omega$ except on the manifold $\Gamma$. Equations $(5 \mathrm{~b})$ and $(5 \mathrm{c})$ respectively express that the displacements at the boundaries of $\Omega^{+}$and $\Omega^{-}$differ by the slip vector $b$, and that the tractions at the boundaries of $\Omega^{+}$and $\Omega^{-}$are in static equilibrium, i.e., equal and opposite. It is to be remarked that this condition corresponds to a standard linear approximation in the small-slip limit, as the traction equilibrium at the fault occurs in fact in the deformed configuration. The boundary conditions (5d) and (5e) correspond to Dirichlet and Neumann boundary conditions, representing a prescribed displacement and a prescribed traction, respectively.

To facilitate the presentation, we note that the solution to the Volterra dislocation problem (5) can be separated into a discontinuous part $u_{0}: \Omega \backslash \Gamma \rightarrow \mathbb{R}^{N}$ with homogeneous data and a continuous part $u_{1}: \Omega \rightarrow \mathbb{R}^{N}$ with inhomogeneous data:

$$
\begin{array}{rlrl}
-\operatorname{div} \sigma\left(u_{0}\right)=0 & & \text { in } \Omega \backslash \Gamma \\
u_{0}=0 & & \text { on } \mathcal{D} \\
\sigma_{n}\left(u_{0}\right)=0 & & \text { on } \mathcal{N} \\
\left.\left[u_{0}\right]\right] & =b & & \text { on } \Gamma \\
\left.\left[\sigma_{\nu}\left(u_{0}\right)\right]\right] & =0 & & \text { on } \Gamma
\end{array}
$$

The sum $u_{0}+u_{1}$ satisfies (5). Therefore, the inhomogeneous data $f, g, h$ in (5) can be treated separately in a standard continuous elasticity problem, and without loss of generality we can restrict our consideration to homogeneous data. We retain $f:=0$ to identify the right member of (5a).

For rupturing faults, some compatibility conditions arise with respect to the boundary conditions. In particular, an intersection of the dislocation with the boundary of the domain is only admissible at the Neumann boundary $\mathcal{N}$. Otherwise, an inadmissible incompatibility between the jump condition $\left[\left[u_{0}\right]\right]=b$ and the homogeneous Dirichlet condition $u_{0}=0$ ensues. 


\subsection{The weak form}

To derive the weak formulation of (5), we note that for any piecewise smooth function $v$ from $\Omega \backslash \Gamma$ into $\mathbb{R}^{N}$, we have the identities:

$$
\begin{aligned}
\int_{\Omega \backslash \Gamma} v \cdot \operatorname{div} \sigma(u)= & \oint_{\partial \Omega^{-}} v \cdot \sigma_{n}(u)-\int_{\Omega^{-}} \sigma(u): \nabla v \\
& +\oint_{\partial \Omega^{+}} v \cdot \sigma_{n}(u)-\int_{\Omega^{+}} \sigma(u): \nabla v \\
= & \oint_{\partial \Omega} v \cdot \sigma_{n}(u)-\int_{\Omega \backslash \Gamma} \sigma(u): \nabla v \\
& +\int_{\Gamma}[[v]] \cdot\left\{\sigma_{\nu}(u)\right\}+\{v\} \cdot\left[\left[\sigma_{\nu}(u)\right]\right]
\end{aligned}
$$

The first identity results from integration by parts. The second identity follows from a rearrangement of the boundary terms and

$$
\begin{aligned}
\int_{\Gamma} v^{+} \cdot \sigma_{n}^{+}(u)+v^{-} \cdot \sigma_{n}^{-}(u)= & \int_{\Gamma} v^{+} \cdot \sigma_{\nu}^{+}(u)-v^{-} \cdot \sigma_{\nu}^{-}(u) \\
= & \int_{\Gamma}\left(v^{+}-v^{-}\right) \frac{1}{2}\left(\sigma_{\nu}^{+}(u)+\sigma_{\nu}^{-}(u)\right) \\
& +\int_{\Gamma} \frac{1}{2}\left(v^{+}+v^{-}\right)\left(\sigma_{\nu}^{+}(u)-\sigma_{\nu}^{-}(u)\right)
\end{aligned}
$$

In the weak formulation, the admissible displacement fields will be insufficiently regular to ensure the existence of the tractions $\sigma_{n}(\cdot)$. Hence, the terms involving these tractions in (7) must be eliminated by means of the boundary conditions and auxiliary conditions on $v$. The traction term on the Neumann boundary can be eliminated by means of (5e). To remove the traction term on $\partial \Omega$, we stipulate that $v$ vanishes on $\mathcal{D}$. The traction average in the final term of $(7)$ is eliminated by requiring that $v$ be continuous. The traction jump in the final term is deleted by means of the traction-continuity condition (5c).

Summarizing, we find that a solution $u$ of (5) satisfies

$$
a_{\Gamma}(u, v)=l(v)
$$

for all sufficiently smooth functions $v: \Omega \rightarrow \mathbb{R}^{N}$ that vanish on $\mathcal{D}$, where

$$
\begin{aligned}
a_{\Gamma}(u, v) & =\int_{\Omega \backslash \Gamma} \sigma(u): \nabla v \\
l(v) & =\int_{\Omega} v \cdot f
\end{aligned}
$$

Note that $v$ is assumed to be smooth on $\Omega$ and, in particular, that it is continuous across the fault $\Gamma$.

To furnish a functional setting for the weak formulation of (5) based on (9), we denote by $\boldsymbol{H}^{k}(\Omega)$ the usual Sobolev space of square-integrable functions from $\Omega$ into $\mathbb{R}^{N}$ with square-integrable distributional derivatives of order $\leq k$, equipped with the inner product

$$
(u, v)_{k, \Omega}=\sum_{|\alpha| \leq k} \int_{\Omega} D^{\alpha} u \cdot D^{\alpha} v
$$


and the corresponding norm $\|\cdot\|_{k, \Omega}$ and semi-norm $|\cdot|_{k, \Omega}$. For the square-integrable functions and the corresponding norm and inner-product, we introduce the condensed notation $\boldsymbol{L}^{2}(\Omega):=\boldsymbol{H}^{0}(\Omega)$, $\|\cdot\|_{\Omega}:=\|\cdot\|_{0, \Omega}$ and $(\cdot, \cdot)_{\Omega}:=(\cdot, \cdot)_{0, \Omega}$. We denote by $\boldsymbol{H}_{0, \mathcal{D}}^{1}(\Omega)$ the subspace of $\boldsymbol{H}^{1}(\Omega)$ of functions that vanish on $\mathcal{D} \subseteq \partial \Omega$. To accommodate the discontinuity corresponding to the dislocation, we introduce the lift operator $\ell_{(\cdot)}$, which assigns to any suitable slip $b: \Gamma \rightarrow \mathbb{R}^{N}$ a function $\ell_{b}$ in $\boldsymbol{H}_{0, \mathcal{D}}^{1}(\Omega \backslash \Gamma)$ such that $\left[\left[\ell_{b}\right]\right]=b$. A precise specification of conditions on the slip distribution is given in Section 4 . The weak formulation of (5) based on (9) writes

Weak formulation: given the lift $\ell_{b} \in \boldsymbol{H}_{0, \mathcal{D}}^{1}(\Omega \backslash \Gamma)$, find $u \in \ell_{b}+\boldsymbol{H}_{0, \mathcal{D}}^{1}(\Omega)$ such that

$$
a_{\Gamma}(u, v)=l(v) \quad \forall v \in \boldsymbol{H}_{0, \mathcal{D}}^{1}(\Omega) .
$$

The bilinear form $a_{\Gamma}: \boldsymbol{H}^{1}(\Omega \backslash \Gamma) \times \boldsymbol{H}^{1}(\Omega \backslash \Gamma) \rightarrow \mathbb{R}$ and linear form $l: \boldsymbol{H}^{1}(\Omega \backslash \Gamma) \rightarrow \mathbb{R}$ in $(11)$, are the extensions (by continuity) of the corresponding forms in 10 . The treatment of the dislocation by means of a lift operator in (11) is analogous to the treatment of inhomogeneous Dirichlet boundary conditions in weak formulations; see, e.g., [7, p.113 ]. Let us note that in the weak formulation (11), we have identified $\left\{u \in \boldsymbol{H}^{1}(\Omega \backslash \Gamma): \llbracket u \rrbracket=0\right\}$ with $\boldsymbol{H}^{1}(\Omega)$. This identification is unambiguous, on account of a one-to-one correspondence between the functions in these spaces.

To analyze the weak formulation (11), and to prepare the presentation of the Weakly-enforcedSlip method in Section 3 , we note that (11) is to be interpreted in the following manner: find $u:=\bar{u}+\ell_{b}$ with $\bar{u} \in \boldsymbol{H}_{0, \mathcal{D}}^{1}(\Omega)$ such that

$$
a(\bar{u}, v)=l(v)-a_{\Gamma}\left(\ell_{b}, v\right) \quad \forall v \in \boldsymbol{H}_{0, \mathcal{D}}^{1}(\Omega) .
$$

where the bilinear form $a: \boldsymbol{H}^{1}(\Omega) \times \boldsymbol{H}^{1}(\Omega) \rightarrow \mathbb{R}$,

$$
a(u, v)=\int_{\Omega} \sigma(u): \nabla v,
$$

corresponds to the restriction of $a_{\Gamma}(\cdot, \cdot)$ to $\boldsymbol{H}^{1}(\Omega) \times \boldsymbol{H}^{1}(\Omega)$. Indeed, for all pairs $(u, v) \in \boldsymbol{H}^{1}(\Omega) \times$ $\boldsymbol{H}^{1}(\Omega)$, the function $\sigma(u): \nabla v$ is Lebesgue integrable on $\Omega$, and because the manifold $\Gamma$ corresponds to a set of $N$-Lebesgue measure zero, the integrals of $\sigma(u): \nabla v$ on $\Omega \backslash \Gamma$ and on $\Omega$ coincide. It is important to note that the restriction of the bilinear form $a_{\Gamma}(\cdot, \cdot)$ to $\boldsymbol{H}^{1}(\Omega) \times \boldsymbol{H}^{1}(\Omega)$ in $(13)$ is independent of the fault $\Gamma$. The function $\bar{u}$ in (11) is referred to as the continuous complement of the solution $u$ with respect to the jump lift $\ell_{b}$ and, indeed, it resides in $\boldsymbol{H}_{0, \mathcal{D}}^{1}(\Omega)$.

For the assumed linear-elastic behavior according to (1) and (2), it follows straightforwardly that

$$
\begin{aligned}
& \left|a_{\Gamma}(u, v)\right| \leq \bar{c}_{A}|u|_{1, \Omega \backslash \Gamma}|v|_{1, \Omega \backslash \Gamma} \\
& \left|a_{\Gamma}(u, u)\right| \geq \underline{c}_{A}|u|_{1, \Omega \backslash \Gamma}^{2}
\end{aligned}
$$

for all $u, v \in \boldsymbol{H}^{1}(\Omega \backslash \Gamma)$, where $\bar{c}_{A}$ and $\underline{c}_{A}$ denote the continuity and strong-positivity constants of the elasticity tensor, respectively, and $|\cdot|_{1, \Omega \backslash \Gamma}$ represents the usual $\boldsymbol{H}^{1}$-seminorm. By virtue of Poincaré's inequality (see, e.g., [2, Theorem 5.3.5]) there exists a bounded positive constant $C_{P}$ such that

$$
\|u\|_{1, \Omega \backslash \Gamma} \leq C_{P}|u|_{1, \Omega \backslash \Gamma} \quad \forall u \in \boldsymbol{H}_{0, \mathcal{D}}^{1}(\Omega \backslash \Gamma)
$$


Hence, the $\boldsymbol{H}^{1}$-norm and $\boldsymbol{H}^{1}$-semi norm are equivalent on $\boldsymbol{H}_{0, \mathcal{D}}^{1}(\Omega)$. Equations 14 and 15 imply that the bilinear forms $a_{\Gamma}(\cdot, \cdot)$ and, accordingly, $a(\cdot, \cdot)$, are continuous and coercive. Moreover, it is easily verified that the linear form $l(\cdot)-a_{\Gamma}\left(\ell_{b}, \cdot\right): \boldsymbol{H}^{1}(\Omega) \rightarrow \mathbb{R}$ in the right member of $(12)$ is continuous. Problem (12) therefore complies with the conditions of the Lax-Milgram lemma (see, for instance, 2, Theorem 2.7.7]) and, hence, it is well posed.

It is interesting to note that 12 allows for a physical interpretation that is very close to Volterra's classical construction for dislocations, popularly known as the 'Volterra knife': to make a cut in the material, displace the two sides and hold them while welding the seam, and finally release the sides so the material assumes its state of self-stressed equilibrium. The initial cut and displacement is represented by the lift $\ell$, which is not in equilibrium and is hence maintained by an external load. The addition of $\bar{u}$ represents the transition to a state of equilibrium, by removing the external load but leaving the displacement intact.

\subsection{Finite element approximation}

Galerkin finite-element approximation methods for Volterra's dislocation problem (11) are generally based on a restriction of the weak formulation to a suitable finite dimensional subspace. The general structure of finite-element methods can be found in many textbooks, for instance, [11, 22, 7, 4]. We present here the main concepts and definitions for the ensuing exposition.

The approximation spaces in finite-element methods are generally subordinate to a mesh $\mathcal{T}_{h}$, viz., a cover of the domain by non-overlapping element domains $\kappa \subset \Omega$. The subscript $h>0$ indicates the dependence of the mesh on a resolution parameter, for instance, the diameter of the largest element in the mesh. In general, we impose some auxiliary conditions on the mesh, such as shape-regularity of the elements and conditions on the connectivity between elements; see, for instance, [7, 5] for further details. A finite-element approximation space $\boldsymbol{V}_{h}^{p} \subset \boldsymbol{H}_{0, \mathcal{D}}^{1}(\Omega)$ subordinate to $\mathcal{T}_{h}$ can then be defined, for instance, as the subspace of vector-valued continuous element-wise polynomials of degree $\leq p$ which vanish on $\mathcal{D}$ :

$$
\boldsymbol{V}_{h}^{p}=\left\{v_{h} \in C^{0}\left(\bar{\Omega}, \mathbb{R}^{N}\right):\left.\left(v_{h}\right)_{i}\right|_{\kappa} \in \mathbb{P}^{p} \text { for all } \kappa \in \mathcal{T}_{h}, i=1,2, \ldots, N,\left.v_{h}\right|_{\mathcal{D}}=0\right\}
$$

with $\mathbb{P}^{p}$ the $N$-variate polynomials of degree $p$. Below, our interest is generally restricted to the $h$-dependence of the approximation space and, accordingly, we will suppress $p$. The finite-element approximation of (11) based on an approximation space $\boldsymbol{V}_{h}$ writes: find $u_{h}:=\bar{u}_{h}+\ell_{b}$ with $\bar{u}_{h} \in \boldsymbol{V}_{h}$ subject to

$$
a\left(\bar{u}_{h}, v_{h}\right)=l\left(v_{h}\right)-a_{\Gamma}\left(\ell_{b}, v_{h}\right) \quad \forall v_{h} \in \boldsymbol{V}_{h} .
$$

We refer the right-most term in the right member of 17 as the lift term.

Approximation properties of the Finite Element Method are generally investigated on the basis of a sequence of meshes $\mathcal{T}_{\mathcal{H}}:=\left(\mathcal{T}_{h}\right)_{h \in \mathcal{H}}$, parametrized by a decreasing sequence of mesh parameters $\mathcal{H}=\left\{h_{1}, h_{2}, \ldots\right\}$ with 0 as only accumulation point. A sequence of meshes is called quasi uniform if there exist positive constants $\underline{C}$ and $\bar{C}$, independent of $h$, such that $\underline{C} h \leq \operatorname{diam}(\kappa) \leq \bar{C} h$ for all $\kappa \in \mathcal{T}_{h}$ and all $h \in \mathcal{H}$. Standard interpolation theory in Sobolev spaces (see, for instance, [7, 2]) conveys that a sequence of approximation spaces $\boldsymbol{V}_{\mathcal{H}}$ of the form $(16)$ based on quasi-uniform meshes possesses the following approximation property: there exists a positive constant $\mathscr{C}$ 用 independent

\footnotetext{
${ }^{\ddagger}$ We use $\mathscr{C}$ to denote a generic positive constant, of which the value and connotation may change from one instance to the next, even within a single chain of expressions.
} 
of $h$ such that for all $h \in \mathcal{H}$, all $k \geq 0$ and both $m \in\{0,1\}$, it holds that

$$
\inf _{v_{h} \in \boldsymbol{V}_{h}}\left\|v-v_{h}\right\|_{m, \Omega} \leq \mathscr{C} h^{l+1-m}|v|_{l+1, \Omega} \quad \forall v \in \boldsymbol{H}^{k+2}(\Omega) \cap \boldsymbol{H}_{0, \mathcal{D}}^{1}(\Omega)
$$

with $l=\min \{p, k+1\}$. The estimate $(18)$ imparts that for all sufficiently smooth $v \in \boldsymbol{H}_{0, \mathcal{D}}^{1}(\Omega)$, the $\|\cdot\|_{m, \Omega}$-norm of the best approximation in $\boldsymbol{V}_{h}$ in that norm decays as $O\left(h^{p+1-m}\right)$ as $h \rightarrow 0$.

The lift $\ell_{b}$ in (17) is in principle arbitrary. However, the use of an arbitrary lift carries severe algorithmic complexity, as one has to explicitly construct the lift and evaluate integrals involving products of (gradients of) the lift and finite-element shape functions. Moreover, the evaluation of these integrals by a suitable numerical integration scheme generally leads to a high computational complexity, because the fault is allowed to intersect elements, and there are no efficient quadrature schemes to integrate the discontinuous function that arises. Therefore, in practice, it is convenient to integrate the lift in the finite-element setting. Provided that that the fault coincides with element edges, a lift $\ell_{b h}$ is then constructed in the broken approximation space:

$$
\hat{\boldsymbol{V}}_{h}=\left\{u \in C^{0}(\bar{\Omega} \backslash \Gamma):\left.\left.u\right|_{\Omega^{ \pm}} \in\left(\boldsymbol{V}_{h}\right)\right|_{\Omega^{ \pm}}\right\}
$$

It is to be noted that the slip does not generally reside in $\left.\left[\hat{\boldsymbol{V}}_{h}\right]\right]$ and, accordingly, $b$ is to be replaced by a suitable interpolant $b_{h}$. Moreover, if the fault does not coincide with element edges, then it is to be replaced by an approximation subject to this condition. The aforementioned approach corresponds to the split-node method by Melosh [14, where the adjective 'split' refers to the discontinuities between the elements in $\Omega^{+}$and $\Omega^{-}$contiguous to $\Gamma$. The split-node approach is analogous to the standard treatment of Dirichlet boundary conditions; see, for instance, [7, §3.2.2]. The split-node approach bypasses the aforementioned complications of an arbitrary-lift approach and the evaluation of the lift term comes essentially free of charge as part of the regular stiffness matrix. A fundamental disadvantage of the split-node approach, however, is that it requires that the fault coincides with element edges, which connects the fault geometry to the geometry and, generally, the topology of the mesh. As a result, computational primitives such as the stiffness matrix and preconditioners for the stiffness matrix, which are contingent on the mesh, cannot be reused for analyses of alternative fault geometries. This is a prohibitive restriction if many dislocation geometries have to be considered, for instance, in inverse problems.

\section{The Weakly-enforced Slip Method}

In section 2.3 we substantiated that the treatment of the lift term in the split-node approach, which is the natural counterpart of the standard treatment of Dirichlet boundary conditions in finiteelement approximations, is unsuitable if many fault geometries have to be analysed, on account of the inherent dependence of the finite-element mesh on the fault geometry.

In this section we propose a new and fundamentally different treatment of the lift term that retains mesh independence: the Weakly-enforced Slip Method (WSM). Below, we present two different formal derivations of the WSM formulation. The derivation in Section 3.1 relies on a limit procedure. In Section 3.2, we derive the WSM formulation on the basis of Nitsche's variational principle for enforcing Dirichlet-type boundary conditions [15]. 


\subsection{Collapsing the lift}

Our aim is to derive a tractable finite-element approximation of Volterra's dislocation problem (11), in which the finite-element space and the bilinear form and, accordingly, the stiffness matrix are independent of the fault geometry.

In principle, the lift-based Galerkin formulation (17) already exhibits the appropriate form. However, as elaborated in Section 2.3. the corresponding finite-element formulation is intractable for general lift operators. One can infer, however, that the complications engendered by a general lift operator can be avoided by collapsing the support of the lift on the fault. The integration of a discontinuous function in $\Omega$ then reduces to the integration of a smooth function on the dislocation. Numerical evaluation of integrals on the dislocation is feasible given a parametrization of the fault. Moreover, the intricate explicit construction of a lift is obviated, and only the slip distribution itself is required, which is presented as part of the problem specification. A further advantage of collapsing the lift is that of localization: instead of having to evaluate the lift term for all shape functions of which the support intersects with the support of the lift, only the shape functions of which the support intersects with the dislocation have to be considered.

To derive the lift term corresponding to a collapsed lift, we consider a symmetric lift $\ell_{b}^{\varepsilon}$ as illustrated in Fig. 2, with compact support in an $\varepsilon$-neighborhood of the fault:

$$
\Gamma^{\varepsilon}:=\{x \in \Omega: \operatorname{dist}(x, \Gamma)<\varepsilon\} .
$$

By virtue of the compact support of $\ell_{b}^{\varepsilon}$ in $\Gamma^{\varepsilon}$, the lift term of 17 evaluates to

$$
a_{\Gamma}\left(\ell_{b}^{\varepsilon}, v\right)=\int_{\Gamma^{\varepsilon} \backslash \Gamma} \sigma(v): \nabla \ell_{b}^{\varepsilon}=\int_{\Gamma} b \cdot\left\{\sigma_{\nu}(v)\right\}-\int_{\Gamma^{\varepsilon} \backslash \Gamma} \ell_{b}^{\varepsilon} \cdot \operatorname{div} \sigma(v) .
$$

The first identity follows from the symmetry of the bilinear form in 10a . The second identity results from integration-by-parts and $\left.\left[\ell_{b}^{\varepsilon}\right]\right]=b$ and $\left\{\ell_{b}^{\varepsilon}\right\}=0$. Let us note that the second identity is formal in the sense that it requires more regularity of $v$ than is actually provided by $\boldsymbol{H}^{1}(\Omega)$. We shall momentarily ignore this aspect, but it manifests itself in the analysis of the approximation properties of the WSM formulation in Section 4. Without loss of generality, we can assume $\ell_{b}^{\varepsilon}$ to be bounded independent of $\varepsilon$. The second term in the final expression in 21) then vanishes if $\Gamma^{\varepsilon}$ collapses on $\Gamma$. Therefore, formally passing to the limit in (21), we obtain

$$
a_{\Gamma}\left(\ell_{b}^{\varepsilon}, v\right) \stackrel{\varepsilon \rightarrow+0}{\longrightarrow} \int_{\Gamma} b \cdot\left\{\sigma_{\nu}(v)\right\}
$$

According to 22 , the lift term reduces to an integral on $\Gamma$ in the limit of collapsing the support of the lift onto the fault. The WSM formulation corresponds to replacing the lift term $a_{\Gamma}\left(\ell_{b}, \cdot\right)$ in the right member of (17) by the limit functional according to (22):

Weakly-enforced Slip Method: given a slip distribution $b: \Gamma \rightarrow \mathbb{R}^{N}$, find $u_{h} \in \boldsymbol{V}_{h}$ such that

$$
a\left(u_{h}, v_{h}\right)=l\left(v_{h}\right)-\int_{\Gamma} b \cdot\left\{\sigma_{\nu}\left(v_{h}\right)\right\} \quad \forall v_{h} \in \boldsymbol{V}_{h} .
$$




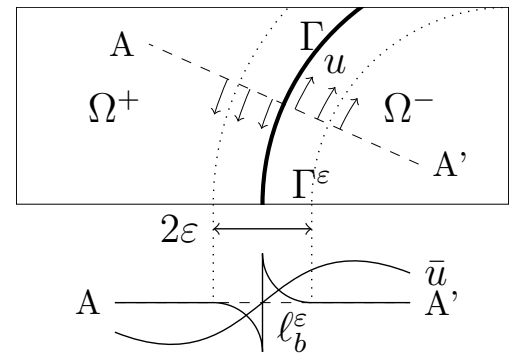

Figure 2: Schematic representation of an $\varepsilon$-local lift $\operatorname{supp}\left(\ell_{b}^{\varepsilon}\right) \in \Gamma^{\varepsilon}$, which is added to a continuous $\bar{u}$ to form the discontinuous displacement field $u$.

The nomenclature Weakly-enforced Slip Method serves to indicate that in (23) the slip discontinuity is weakly enforced in the right-hand side only, and does not appear in the approximation space.

It is to be noted that although the WSM formulation 23 is derived from the lift-based formulation (17) by collapsing the lift, in contrast to (17) we do not add a lift to the continuous complement $u_{h}$. Because $\boldsymbol{V}_{h} \subset C^{0}\left(\Omega, \mathbb{R}^{N}\right)$, WSM thus yields a continuous approximation to the discontinuous solution of the Volterra dislocation problem (11). This implies that the approximation near the dislocation will inevitably be inaccurate. We will however show in Section 4 that away from the dislocation, the error in the WSM approximation converges optimally under mesh refinement.

\subsection{Alternative derivation via Nitsche's variational principle}

To further elucidate the WSM formulation, we present in this section an alternative derivation of (23) based on Nitsche's Variationsprinzip [15. Nitsche presented in [15] a variational principle for weakly imposing Dirichlet boundary conditions in finite-element approximations of elliptic problems, i.e., without incorporating such essential boundary conditions in the approximation space. Nitsche's variational principle can be extended to the Volterra dislocation problem (11) to weakly impose the slip discontinuity. To specify this extension, we consider a suitable broken space $\hat{\boldsymbol{V}}(h)$ which encapsulates the broken approximation space $\hat{\boldsymbol{V}}_{h}$ and contains the solution $u$ to the Volterra dislocation problem (11). We define the quadratic functional $J: \hat{\boldsymbol{V}}(h) \rightarrow \mathbb{R}$ :

$$
J(w)=\frac{1}{2} a_{\Gamma}(w, w)-\int_{\Gamma}[[w]] \cdot\left\{\sigma_{\nu}(w)\right\}+\frac{\psi}{2} \int_{\Gamma}[[w]]^{2},
$$

for some suitable constant $\psi>0$, generally dependent on $h$. Let $\check{\boldsymbol{V}}_{h}$ denote either the broken approximation space $\hat{\boldsymbol{V}}_{h}$ or the continuous approximation space $\boldsymbol{V}_{h}$ and consider the approximation $\check{u}_{h} \approx u$ according to:

$$
\check{u}_{h}:=\underset{v_{h} \in \check{\boldsymbol{V}}_{h}}{\arg \inf } J\left(u-v_{h}\right)
$$

Equation 25 implies that $\check{u}_{h}$ satisfies the Kuhn-Tucker optimality condition $J^{\prime}\left(u-\check{u}_{h}\right)\left(v_{h}\right)=0$ for all $v_{h} \in \boldsymbol{V}_{h}$, where $v \mapsto J^{\prime}(w)(v)$ denotes the Fréchet derivative of $J$ at $w$. For $J$ according 
to (24), the optimality condition implies that $\check{u}_{h} \in \check{\boldsymbol{V}}_{h}$ satisfies:

$$
\begin{aligned}
& \left.a_{\Gamma}\left(\check{u}_{h}, v_{h}\right)-\int_{\Gamma}\left[\left[\check{u}_{h}\right]\right] \cdot\left\{\sigma_{\nu}\left(v_{h}\right)\right\}-\int_{\Gamma}\left[\left[v_{h}\right]\right] \cdot\left\{\sigma_{\nu}\left(\check{u}_{h}\right)\right\}+\psi \int_{\Gamma}\left[\left[\check{u}_{h}\right]\right] \cdot \llbracket\left[v_{h}\right]\right] \\
& \quad=a_{\Gamma}\left(u, v_{h}\right)-\int_{\Gamma}[[u]] \cdot\left\{\sigma_{\nu}\left(v_{h}\right)\right\}-\int_{\Gamma}\left[\left[v_{h}\right]\right] \cdot\left\{\sigma_{\nu}(u)\right\}+\psi \int_{\Gamma}\left[[u] \cdot[[v]]_{h}\right. \\
& \quad=l\left(v_{h}\right)-\int_{\Gamma} b \cdot\left\{\sigma_{\nu}\left(v_{h}\right)\right\}+\psi \int_{\Gamma} b \cdot \llbracket[v]_{h} \quad \forall v_{h} \in \check{\boldsymbol{V}}_{h} .
\end{aligned}
$$

The final identity follows by invoking integration-by-parts on $a_{\Gamma}\left(u, v_{h}\right)$, a rearrangement of terms and the strong formulation of Volterra's dislocation problem in (5).

If the broken approximation space $\hat{\boldsymbol{V}}_{h}$ is inserted for $\check{\boldsymbol{V}}_{h}$, the optimality condition (26) can be reinterpreted as a symmetric-interior-penalty (SIP) discontinuous-Galerkin-type formulation; see, for instance, [5, Sec. 4.2]. In contrast to standard discontinuous Galerkin formulations, the slip terms in the right-hand side, i.e., the terms containing $b$ in the ultimate expression in (26), enforce the jump discontinuity at the fault. Convergence results for this formulation can be established in a similar manner as in [15]. For suitable stabilization parameters $\psi$, the functional $J$ in (24) is equivalent to $\|\cdot\|_{1, \Omega \backslash \Gamma}$ and (25) implies quasi-optimal convergence of $\check{u}_{h}$.

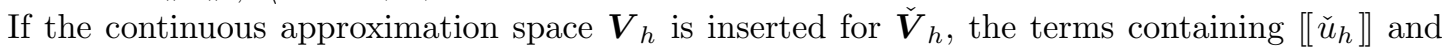
[[v $\left.v_{h}\right]$ vanish, and we obtain the WSM formulation (23). Hence, WSM can indeed be interpreted as an extension of Nitsche's variational principle to the Volterra dislocation problem with continuous approximation spaces. Furthermore, in view of $\hat{\boldsymbol{V}}_{h} \supset \boldsymbol{V}_{h}$, WSM can also be regarded as a SIP discontinuous Galerkin formulation, based on a continuous subspace. One can infer that the WSM approximation retains the quasi-optimal approximation property in $\|\cdot\|_{1, \Omega \backslash \Gamma}$. However, since the continuous approximation spaces applied in WSM are not dense in $H_{0, \mathcal{D}}^{1}(\Omega \backslash \Gamma)$, the immediate significance of this quasi-optimality for the approximation properties of WSM is limited.

\section{Approximation properties of WSM}

An analysis of the approximation properties of the Weakly-enforced Slip Method is non-trivial, owing to the fact that in WSM one considers approximations in $\boldsymbol{H}_{0, \mathcal{D}}^{1}(\Omega)$-conforming subspaces, while the solution itself resides in $\boldsymbol{H}_{0, \mathcal{D}}^{1}(\Omega \backslash \Gamma)$, and the embedding of $\boldsymbol{H}_{0, \mathcal{D}}^{1}(\Omega)$ in $\boldsymbol{H}_{0, \mathcal{D}}^{1}(\Omega \backslash \Gamma)$ is non-dense. Essentially, we attempt to approximate a discontinuous function by a continuous one and, in doing so, we incur an error that does not vanish under mesh refinement. Standard techniques to assess global approximation properties, on all of $\Omega \backslash \Gamma$, viz., Céa's lemma or the Strang lemmas [7, Lems. 2.25-27], therefore provide only partial information; see Section 4.2 .

To provide a foundation for analyzing the approximation provided by WSM, we we first recall some aspects of traces and tractions in Section 4.1. Section 4.2 investigates the global approximation properties of WSM, i.e., on the entire domain. Section 4.3 establishes the local approximation properties of WSM, i.e, on the domain excluding a neighborhood of the fault.

\subsection{Traces and tractions}

To enable an analysis of the approximation behavior of WSM, some elementary aspects of trace theory are required. For a comprehensive overview, we refer to [18. To make the theory applicable to the Volterra dislocation problem, we must impose auxiliary smoothness conditions on the 
elasticity tensor. In particular, we assume:

$$
A_{i j k l} \in C^{1}(\bar{\Omega})
$$

It is to be noted that (27) implies that the elasticity tensor is $C^{1}$ continuous on the domain, including the boundary, and across the fault, including the dislocation. The $C^{1}$ continuity on the subdomains $\overline{\Omega^{+}}$and $\overline{\Omega^{-}}$ensures that tractions are well defined. The $C^{1}$ continuity across the fault is required to establish global convergence of the WSM approximation in the $\boldsymbol{L}^{2}$-norm and local convergence in the $\boldsymbol{H}^{1}$-norm; see Sections 4.2 and 4.3 below. Let us note that in tectonophysics the elasticity tensor is generally assumed to be uniformly constant in the domain.

Let $\omega \subset \mathbb{R}^{N}$ denote an arbitrary connected domain with Lipschitz boundary. In particular, recalling the partition of $\Omega$ into the complementary subsets $\Omega^{ \pm}$, we envisage $\omega \in\left\{\Omega^{+}, \Omega^{-}\right\}$. We denote by $\widehat{\gamma}$ the restriction of a function in $\boldsymbol{C}^{1}(\bar{\omega})$ to the boundary $\partial \omega$. By virtue of the density of $\boldsymbol{C}^{1}(\omega)$ in $\boldsymbol{H}^{1}(\omega)$, the operator can be extended to a linear continuous trace operator, denoted by $\gamma$, from $\boldsymbol{H}^{1}(\omega)$ into $\boldsymbol{L}^{2}(\partial \omega)$. The image of $\gamma$ is denoted by $\boldsymbol{H}^{1 / 2}(\partial \omega)$. Considering a subset $\varkappa \subset \partial \omega$, we denote by $\gamma_{\varkappa}:=\left.(\gamma(\cdot))\right|_{\varkappa}$ the composition of the trace operator and the restriction to $\varkappa$. The image of $\gamma_{\varkappa}$ restricted to the class of functions $\boldsymbol{H}_{0, \partial \omega \backslash \varkappa}^{1}(\omega)$ that vanish on $\partial \omega \backslash \varkappa$ is indicated by $\boldsymbol{H}_{0}^{1 / 2}(\varkappa)$ :

$$
\boldsymbol{H}_{0}^{1 / 2}(\varkappa)=\left\{\gamma_{\varkappa} u: u \in \boldsymbol{H}_{0, \partial \omega \backslash \varkappa}^{1}(\omega)\right\} .
$$

The space $\boldsymbol{H}_{0}^{1 / 2}(\varkappa)$ can be endowed with the norm:

$$
\|\lambda\|_{1 / 2, \varkappa}:=\inf \left\{\|u\|_{1, \omega}: u \in \boldsymbol{H}_{0, \partial \omega \backslash \varkappa}^{1}(\omega), \gamma_{\varkappa} u=\lambda\right\} .
$$

with the obvious extension to $\boldsymbol{H}^{1 / 2}(\partial \omega)$. There exist continuous right inverses

$$
\gamma^{-1}: \boldsymbol{H}^{1 / 2}(\partial \omega) \rightarrow \boldsymbol{H}^{1}(\omega), \quad \gamma_{\varkappa}^{-1}: \boldsymbol{H}_{0}^{1 / 2}(\varkappa) \rightarrow \boldsymbol{H}_{0, \partial \omega \backslash \varkappa}^{1}(\omega),
$$

of $\gamma$ and $\gamma_{\varkappa}$. Such a right inverse is called a lifting (or lift) of the trace. It is to be noted that lift operators are generally non-unique.

We denote by $\widehat{\sigma}_{n}: u \mapsto n \cdot \gamma(\sigma(u))$ the traction of a function in $\boldsymbol{C}^{2}(\bar{\omega})$ on $\partial \omega$, where $n$ denotes the exterior unit normal vector on $\partial \omega$. We define

$$
\boldsymbol{H}_{\operatorname{div} \sigma}^{1}(\omega):=\left\{u \in \boldsymbol{H}^{1}(\omega): \operatorname{div} \sigma(u) \in \boldsymbol{L}^{2}(\omega)\right\} .
$$

Applying index notation for transparency, the chain rule yields

$$
\partial_{j} \sigma_{i j}(u)=\left(\partial_{j} A_{i j k l}\right) \epsilon_{k l}(u)+A_{i j k l}\left(\partial_{j} \epsilon_{k l}(u)\right)
$$

Therefore, the condition $\operatorname{div} \sigma(u) \in \boldsymbol{L}^{2}(\omega)$ provides a meaningful condition on $u$ if $\partial_{j} A_{i j k l} \in L^{\infty}(\omega)$. For $\omega \in\left\{\Omega^{+}, \Omega^{-}\right\}$, this auxiliary condition on the elasticity tensor is satisfied under the standing assumption 27). The vector space $\boldsymbol{H}_{\operatorname{div} \sigma}^{1}(\omega)$ is a Hilbert space under the inner-product associated with the norm $\left(\|\cdot\|_{1, \omega}^{2}+\|\operatorname{div} \sigma(\cdot)\|_{\omega}^{2}\right)^{1 / 2}$. The traction $\widehat{\sigma}_{n}$ can be extended to a bounded linear operator, denoted by $\sigma_{n}$, from $\boldsymbol{H}_{\operatorname{div} \sigma}^{1}(\omega)$ into $\boldsymbol{H}^{-1 / 2}(\partial \omega):=\left[\boldsymbol{H}^{1 / 2}(\partial \omega)\right]^{\prime}$, the dual space of $\boldsymbol{H}^{1 / 2}(\partial \omega)$. For each $u \in \boldsymbol{H}_{\operatorname{div} \sigma}^{1}(\omega)$, the functional $\sigma_{n}(u)$ acts on functions in $\boldsymbol{H}^{1 / 2}(\partial \omega)$ by means of the following duality pairing:

$$
\left\langle\sigma_{n}(u), \lambda\right\rangle=\int_{\omega} \operatorname{div} \sigma(u) \cdot \gamma^{-1}(\lambda)+\int_{\omega} \sigma(u): \nabla \gamma^{-1}(\lambda)
$$


One may note that for functions in $C^{2}(\bar{\omega})$, Equation 33 corresponds to a standard integrationby-parts identity. Continuity of the operator $\sigma_{n}$ thus defined follows from the sequence of bounds:

$$
\begin{aligned}
\left|\left\langle\sigma_{n}(u), \lambda\right\rangle\right| & \leq\|\operatorname{div} \sigma(u)\|_{\omega}\left\|\gamma^{-1}(\lambda)\right\|_{\omega}+\|\sigma(u)\|_{\omega}\left|\gamma^{-1}(\lambda)\right|_{1, \omega} \\
& \leq\left(\|\operatorname{div} \sigma(u)\|_{\omega}^{2}+\|\sigma(u)\|_{\omega}^{2}\right)^{1 / 2}\left(\left\|\gamma^{-1}(\lambda)\right\|_{\omega}^{2}+\left|\gamma^{-1}(\lambda)\right|_{1, \omega}\right)^{1 / 2} \\
& \leq\left(1+\bar{c}_{A}^{2}\right)^{1 / 2}\left(\|u\|_{1, \omega}^{2}+\|\operatorname{div} \sigma(u)\|_{\omega}^{2}\right)^{1 / 2}\left\|\gamma^{-1}(\lambda)\right\|_{1, \omega}
\end{aligned}
$$

and the continuity of the lifting of the trace from $\boldsymbol{H}^{1 / 2}(\partial \omega)$ into $\boldsymbol{H}^{1}(\omega)$. The dual space $\left[\boldsymbol{H}^{1 / 2}(\partial \omega)\right]^{\prime}$ is a Banach space under the norm

$$
\|v\|_{-1 / 2, \partial \omega}=\sup _{\lambda \in \boldsymbol{H}^{1 / 2}(\partial \omega)} \frac{\langle v, \lambda\rangle}{\|\lambda\|_{1 / 2, \partial \omega}} .
$$

The restriction of the traction $\left.\left(\widehat{\sigma}_{n}(\cdot)\right)\right|_{\varkappa}$ to a subset $\varkappa \subset \partial \omega$ of the boundary can be extended to a bounded linear operator $\sigma_{n} \varkappa$ from $\boldsymbol{H}_{\text {div } \sigma}^{1}(\omega)$ into $\boldsymbol{H}^{-1 / 2}(\varkappa):=\left[\boldsymbol{H}_{0}^{1 / 2}(\varkappa)\right]^{\prime}$. The functional $\sigma_{n, \varkappa}(u)$ acts on functions in $\boldsymbol{H}_{0}^{1 / 2}(\varkappa)$ via the duality pairing:

$$
\left\langle\sigma_{n, \varkappa}(u), \lambda\right\rangle=\int_{\omega} \operatorname{div} \sigma(u) \cdot \gamma_{\varkappa}^{-1}(\lambda)+\int_{\omega} \sigma(u): \nabla \gamma_{\varkappa}^{-1}(\lambda)
$$

Continuity of the operator $\sigma_{n, \varkappa}$ thus defined follows in a similar manner as in (34).

For non-rupturing faults, it holds that $b \in \boldsymbol{H}_{0}^{1 / 2}(\Gamma)$ and the above definitions apply without revisions. The slip discontinuity $(5 \mathrm{~b})$ and the traction discontinuity $(5 \mathrm{c})$ are then to be understood in the sense of traces and tractions outlined above. However, for rupturing faults, i.e., if the dislocation intersects with the boundary of the domain, then $b \notin \boldsymbol{H}_{0}^{1 / 2}(\Gamma)$, and further consideration is required. We can accommodate $b$ in the space:

$$
\widetilde{\boldsymbol{H}}^{1 / 2}(\Gamma):=\left\{\gamma_{\Gamma} u: u \in \boldsymbol{H}^{1}(\omega)\right\}
$$

with $\omega \in\left\{\Omega^{+}, \Omega^{-}\right\}$. The space $\widetilde{\boldsymbol{H}}^{1 / 2}(\Gamma)$ is a Banach space under the norm

$$
{\widetilde{\|\lambda\|_{1 / 2, \Gamma}}}=\inf \left\{\|u\|_{1, \omega}: u \in \boldsymbol{H}^{1}(\omega), \gamma_{\Gamma} u=\lambda\right\}
$$

The principal complication pertaining to rupturing faults, is that the corresponding slip vectors cannot be lifted into $\boldsymbol{H}_{0, \partial \omega \backslash \Gamma}^{1}(\omega)$, as traces of functions in $\boldsymbol{H}^{1}(\omega)$ do not admit the discontinuity that would otherwise arise at the intersection of $\bar{\Gamma}$ and $\overline{\partial \omega \backslash \Gamma}$. Hence, we cannot use (35) to define an extension of the restriction of the traction, $\left.\left(\widehat{\sigma}_{n}(\cdot)\right)\right|_{\Gamma}$, to a bounded linear operator from $\boldsymbol{H}_{\text {div } \sigma}^{1}(\omega)$ into $\left[\widetilde{\boldsymbol{H}}^{1 / 2}(\Gamma)\right]^{\prime}$. However, there exists a continuous right inverse $\widetilde{\gamma}_{\Gamma}^{-1}: \widetilde{\boldsymbol{H}}^{1 / 2}(\Gamma) \rightarrow \boldsymbol{H}^{1}(\omega)$ of the operator $\gamma_{\Gamma}$, for instance,

$$
\widetilde{\gamma}_{\Gamma}^{-1}(\lambda)=\arg \inf \left\{|u|_{1, \omega}: u \in \boldsymbol{H}^{1}(\omega), \gamma_{\Gamma} u=\lambda\right\}
$$

Let us note that the image of the lift operator $\widetilde{\gamma}_{\Gamma}^{-1}$ corresponds to a harmonic function subject to inhomogeneous Dirichlet conditions on $\Gamma$ with data $\lambda$ and a homogeneous Neumann condition on $\partial \omega \backslash \Gamma$. The lift operator $\widetilde{\gamma}_{\Gamma}^{-1}$ can be modified to include homogeneous Dirichlet conditions on 
$\mathcal{D} \subset \partial \omega \backslash \Gamma$ in the codomain, if necessary. The lift operator $\widetilde{\gamma}_{\Gamma}^{-1}$ enables us to extend $\left.\left(\widehat{\sigma}_{n}\right)\right|_{\Gamma}$ to a continuous linear operator:

$$
\widetilde{\sigma}_{n, \Gamma}:\left\{u \in \boldsymbol{H}_{\operatorname{div} \sigma}^{1}(\omega):\left\|\sigma_{n, \partial \omega \backslash \Gamma}(u)\right\|_{-1 / 2, \partial \omega \backslash \Gamma}=0\right\} \rightarrow\left[\widetilde{\boldsymbol{H}}^{1 / 2}(\Gamma)\right]^{\prime}
$$

The functional $\widetilde{\sigma}_{n, \Gamma}(u)$ acts on functions in $\widetilde{\boldsymbol{H}}^{1 / 2}(\Gamma)$ via the duality pairing:

$$
\left\langle\widetilde{\sigma}_{n, \Gamma}(u), \lambda\right\rangle=\int_{\omega} \operatorname{div} \sigma(u) \cdot \widetilde{\gamma}_{\Gamma}^{-1}(\lambda)+\int_{\omega} \sigma(u): \nabla \widetilde{\gamma}_{\Gamma}^{-1}(\lambda)
$$

Essentially, in (39) and (40), we have defined the extension $\widetilde{\sigma}_{n, \Gamma}$ of the restriction of the traction to the dislocation, $\left.\left(\widehat{\sigma}_{n}(\cdot)\right)\right|_{\Gamma}$, by restricting the domain of the extended operator to functions for which the traction vanishes on $\partial \omega \backslash \Gamma$. This restriction in the definition is consistent with the standing assumption that an intersection of the dislocation with the boundary of the domain can only occur at Neumann boundaries.

In the analysis below, we restrict ourselves to non-rupturing faults. The analysis in Section 4.2 however extends to rupturing faults by replacing the spaces and trace and traction operators for non-rupturing faults with those for rupturing faults.

\subsection{Global approximation properties of WSM}

To assess the global approximation properties of WSM, we first construct an upper bound on the functional $\left(b,\left\{\sigma_{\nu}(\cdot)\right\}\right)_{\Gamma}: \boldsymbol{V}_{h} \rightarrow \mathbb{R}$ in the right member of the WSM formulation. It is to be noted that, in general, $\boldsymbol{V}_{h} \not \subset \boldsymbol{H}_{\text {div } \sigma}^{1}(\Omega)$. Hence, the functional $\left(b,\left\{\sigma_{\nu}(\cdot)\right\}\right)_{\Gamma}: \boldsymbol{V}_{h} \rightarrow \mathbb{R}$ does not admit an interpretation as a duality pairing according to 35 . Because $\boldsymbol{V}_{h}$ is piecewise polynomial, however, an upper bound can be constructed on the basis of inverse and trace inequalities. We refer to [5] for a comprehensive treatment of this subject. Inverse and trace inequalities can generally be derived under suitable (sufficient) regularity conditions on the finite-element mesh; see [5, Chap. 1]. A detailed treatment of the conditions underlying inverse and trace inequalities is beyond the scope of this work. Instead, we shall directly assume that a suitable discrete trace inequality holds. To formulate the assumption, for a given sequence of partitions $\mathcal{T}_{\mathcal{H}}$ and for each $h \in \mathcal{H}$, we denote by $\mathcal{S}_{h}$ a dense cover of the fault by means of open intersections of the fault with element interiors or with element faces, i.e.,

$$
\begin{aligned}
\mathcal{S}_{h}= & \left\{s \subset \Gamma: s=\Gamma \cap \kappa \neq \emptyset \text { for some } \kappa \in \mathcal{T}_{h}\right\} \\
& \cup\left\{s \subset \Gamma: s=\operatorname{int}\left(\Gamma \cap \partial \kappa_{0} \cap \partial \kappa_{1}\right) \neq \emptyset \text { for some } \kappa_{0}, \kappa_{1} \in \mathcal{T}_{h}, \kappa_{0} \neq \kappa_{1}\right\}
\end{aligned}
$$

See Figure 3 for an illustration. The separate treatment of element boundaries serves to ensure that subsets of $\Gamma$ that coincide with element faces are separately included in $\mathcal{S}_{h}$. To each segment $s \in \mathcal{S}_{h}$, we associate a pair of contiguous elements $\left\{\kappa_{s}^{+}, \kappa_{s}^{-}\right\}$such that $s \subset \kappa^{ \pm} \cup \partial \kappa^{ \pm}$and $\kappa^{ \pm} \cap \Omega^{ \pm} \neq \emptyset$. If $s \subset \partial \kappa$ (resp. $s \subset \kappa$ ) for some $\kappa$ then $\kappa^{+}$and $\kappa^{-}$will be distinct (resp. identical). We assume that the following discrete trace inequality holds for all $h \in \mathcal{H}$, all $s \in \mathcal{S}_{h}$ and all element-wise polynomial functions $v_{h}$ of degree at most $p$ :

$$
(\operatorname{diam}(\kappa))^{1 / 2}\left\|v_{h}\right\|_{s} \leq C_{\Gamma}\left\|v_{h}\right\|_{\kappa},
$$

for both $\kappa \in\left\{\kappa_{s}^{+}, \kappa_{s}^{-}\right\}$, for some $C_{\Gamma}>0$ independent of $h$; cf. [5, Lemma 1.46]. The constant $C_{\Gamma}$ is allowed to increase with the polynomial order $p$. 


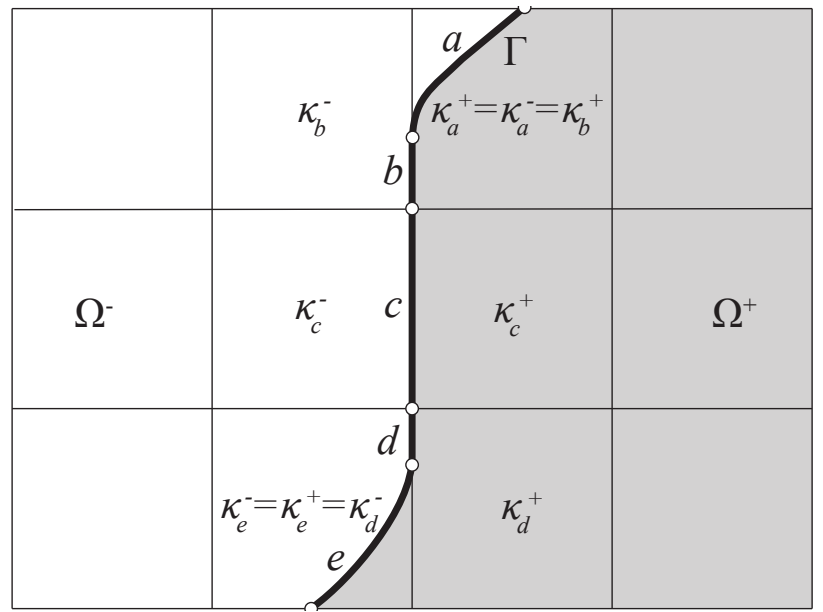

Figure 3: Illustration of the covering of the fault $\Gamma$ by segments $\mathcal{S}_{h}=\{a, b, c, b, e\}$, and the corresponding elements $\left\{\kappa_{(\cdot)}^{+}, \kappa_{(\cdot)}^{-}\right\}$. Because $b, c, d$ coincide with element boundaries $\kappa_{(\cdot)}^{+} \neq \kappa_{(\cdot)}^{-}$for these segments. The segments $a, e$ are interior to elements and, accordingly $\kappa_{(\cdot)}^{+}=\kappa_{(\cdot)}^{-}$for these segments.

Lemma 1 (Continuity of the WSM linear form). Consider a manifold $\Gamma \subset \Omega \subset \mathbb{R}^{N}$, a slip distribution $b \in \boldsymbol{H}_{0}^{1 / 2}(\Gamma)$ and a sequence of partitions $\mathcal{T}_{\mathcal{H}}$ such that for all $h \in \mathcal{H}$, the discrete trace inequality (42) holds for all $s \in \mathcal{S}_{h}$ and all element-wise polynomial functions on $\mathcal{T}_{h}$ and

$$
\|b\|_{\mathcal{T}_{h}, \Gamma}:=\left(\sum_{s \in \mathcal{S}_{h}} h_{s}^{-1}\|b\|_{s}^{2}\right)^{1 / 2}<\infty
$$

with $h_{s}$ the harmonic average of the diameters of the elements adjacent to $s$,

$$
\frac{1}{h_{s}}=\frac{1}{\operatorname{diam}\left(\kappa_{s}^{+}\right)}+\frac{1}{\operatorname{diam}\left(\kappa_{s}^{-}\right)}
$$

Then for all $h \in \mathcal{H}$, the linear form $\left(b,\left\{\sigma_{\nu}(\cdot)\right\}\right)_{\Gamma}: \boldsymbol{V}_{h} \rightarrow \mathbb{R}$ is continuous and

$$
\left|\left(b,\left\{\sigma_{\nu}(v)\right\}\right)_{\Gamma}\right| \leq 2^{-1 / 2} \bar{c}_{A} C_{\Gamma} M^{1 / 2}\|b\|_{\mathcal{T}_{h}, \Gamma}\|v\|_{1, \Omega}
$$

with $\bar{c}_{A}$ the continuity constant of the elasticity tensor in $\left.\sqrt{3}\right), C_{\Gamma}$ the constant in the discrete trace inequality (42) and $M$ the maximum multiplicity of the multiset $\left\{\kappa \in\left\{\kappa_{s}^{+}, \kappa_{s}^{-}\right\}: s \in \mathcal{S}_{h}\right\}$.

Remark. The maximum multiplicity $M$ in Lemma 1 indicates the maximum number of occurrences of any one element in connection to any segment $s \in \mathcal{S}_{h}$ as a member of the set $\left\{\kappa_{s}^{+}, \kappa_{s}^{-}\right\}$. For instance, in Figure 3, the element $\kappa_{a}^{+}=\kappa_{a}^{-}=\kappa_{b}^{+}$has multiplicity 3. One can infer that $M$ is bounded by the maximum number of faces of any element in the mesh, increased by 2 for interior segments.

Proof. We first separate the integral on $\Gamma$ into a sum of contributions from the segments and 
apply (3) and the Cauchy-Schwarz inequality to obtain

$$
\begin{aligned}
\left|\left(b,\left\{\sigma_{\nu}\left(v_{h}\right)\right\}\right)_{\Gamma}\right| & =\left|2^{-1} \sum_{s \in S_{h}}\left(b, \nu \cdot \gamma_{\Gamma}^{+}\left(\sigma\left(v_{h}\right)\right)+\nu \cdot \gamma_{\Gamma}^{-}\left(\sigma\left(v_{h}\right)\right)\right)_{s}\right| \\
& \leq 2^{-1} \bar{c}_{A} \sum_{s \in S_{h}}\|b\|_{s}\left(\left\|\gamma_{\Gamma}^{+}\left(\nabla v_{h}\right)\right\|_{s}+\left\|\gamma_{\Gamma}^{-}\left(\nabla v_{h}\right)\right\|_{s}\right)
\end{aligned}
$$

where $\gamma_{\Gamma}^{ \pm}(\cdot)$ denotes the trace of $(\cdot)$ from within $\Omega^{ \pm}$. Noting that $v_{h} \in \boldsymbol{V}_{h}$ is element-wise polynomial, we deduce from the discrete trace inequality (42), the arithmetic-geometric mean inequality and (44):

$$
\begin{aligned}
\left|\left(b,\left\{\sigma_{\nu}\left(v_{h}\right)\right\}\right)_{\Gamma}\right| & \leq 2^{-1} \bar{c}_{A} C_{\Gamma} \sum_{s \in S_{h}} h_{s}^{-1 / 2}\|b\|_{s}\left(\left\|\nabla v_{h}\right\|_{\kappa_{s}^{+}}+\left\|\nabla v_{h}\right\|_{\kappa_{s}^{-}}\right) \\
& \leq 2^{-1 / 2} \bar{c}_{A} C_{\Gamma}\left(\sum_{s \in S_{h}} h_{s}^{-1}\|b\|_{s}^{2}\right)^{1 / 2}\left(\sum_{s \in S_{h}}\left\|\nabla v_{h}\right\|_{\kappa_{s}^{+}}^{2}+\left\|\nabla v_{h}\right\|_{\kappa_{s}^{-}}^{2}\right)^{1 / 2} \\
& \leq 2^{-1 / 2} \bar{c}_{A} C_{\Gamma} M^{1 / 2}\|b\|_{\mathcal{T}_{h}, \Gamma}\left\|v_{h}\right\|_{1, \Omega}
\end{aligned}
$$

To determine the global approximation properties of the WSM formulation in the $\boldsymbol{H}^{1}$-norm, we note that the WSM formulation is inconsistent: The solution of the weak formulation of the Volterra dislocation problem (11) violates the WSM weak form (23) by $\left(b,\left\{\sigma_{\nu}(v)\right\}\right)_{\Gamma}$, for all $v \in \boldsymbol{V}_{h}$. The second Strang lemma [7, Lemma 2.25] then provides the following characterization of the global approximation properties of WSM:

Theorem 2 (Global approximation properties of WSM in the $\boldsymbol{H}^{1}$-norm). Assume that the conditions of Lemma 1 hold. Let $u \in \boldsymbol{H}_{0, \mathcal{D}}^{1}(\Omega \backslash \Gamma)$ denote the solution to the Volterra dislocation problem (11) and let $u_{h} \in \boldsymbol{V}_{h}$ denote its WSM approximation according to (23). It holds that

$$
\begin{aligned}
\left\|u-u_{h}\right\|_{1, \Omega \backslash \Gamma} \leq & \left(1+C_{P} \underline{c}_{A}^{-1} \bar{c}_{A}\right) \inf _{v_{h} \in \boldsymbol{V}_{h}}\left\|u-v_{h}\right\|_{1, \Omega \backslash \Gamma} \\
& +C_{P} \underline{c}_{A}^{-1} \sup _{v_{h} \in \boldsymbol{V} \backslash\{0\}} \frac{\left|\left(b,\left\{\sigma_{\nu}\left(v_{h}\right)\right\}\right)_{\Gamma}\right|}{\left\|v_{h}\right\|_{1, \Omega}}
\end{aligned}
$$

Proof. We first recall that the bilinear form $a_{\Gamma}(\cdot, \cdot)$ is bounded on $\boldsymbol{H}_{0, \mathcal{D}}^{1}(\Omega \backslash \Gamma) \times \boldsymbol{V}_{h}$ and coercive on $\boldsymbol{V}_{h} \times \boldsymbol{V}_{h}$; see (14) and (15). For arbitrary $v_{h} \in \boldsymbol{V}_{h}$, we have the chain of inequalities:

$$
\begin{aligned}
\left\|u_{h}-v_{h}\right\|_{1, \Omega \backslash \Gamma}^{2} & \leq C_{P} \underline{c}_{A}^{-1}\left|a_{\Gamma}\left(u_{h}-v_{h}, u_{h}-v_{h}\right)\right| \\
& =C_{P} \underline{c}_{A}^{-1}\left|a_{\Gamma}\left(u_{h}-u, u_{h}-v_{h}\right)+a_{\Gamma}\left(u-v_{h}, u_{h}-v_{h}\right)\right| \\
& \leq C_{P} \underline{c}_{A}^{-1}\left(\left|\left(b,\left\{\sigma_{\nu}\left(u_{h}-v_{h}\right)\right\}\right)_{\Gamma}\right|+\bar{c}_{A}\left|u-v_{h}\right|_{1, \Omega \backslash \Gamma}\left|u_{h}-v_{h}\right|_{1, \Omega \backslash \Gamma}\right)
\end{aligned}
$$

which leads to

$$
\left\|u_{h}-v_{h}\right\|_{1, \Omega \backslash \Gamma} \leq C_{P} \underline{c}_{A}^{-1} \sup _{w_{h} \in \boldsymbol{V} h \backslash\{0\}} \frac{\left|\left(b,\left\{\sigma_{\nu}\left(w_{h}\right)\right\}\right)_{\Gamma}\right|}{\left\|w_{h}\right\|_{1, \Omega}}+C_{P} \underline{c}_{A}^{-1} \bar{c}_{A}\left\|u-v_{h}\right\|_{1, \Omega \backslash \Gamma}
$$

The bound 48 then follows from the triangle inequality. 
For quasi-uniform meshes, Lemma 1 and Theorem 2 lead to a simple asymptotic characterization of the global approximation properties of WSM in the $\boldsymbol{H}^{1}$-norm. This characterization is detailed in the following corollary:

Corollary 3. Assume that the conditions of Lemma 1 hold and that the sequence of partitions $\mathcal{T}_{\mathcal{H}}$ is quasi-uniform with respect to the mesh parameter, i.e., for all $h \in \mathcal{H}$ there exist constants $\bar{C}>0$ and $\underline{C}>0$ independent of $h$ such that $\underline{C} h \leq \operatorname{diam}(\kappa) \leq \bar{C} h$ for all $\kappa \in \mathcal{T}_{h}$. It then holds that

$$
\left\|u-u_{h}\right\|_{1, \Omega \backslash \Gamma} \leq \mathscr{C} h^{-1 / 2}
$$

as $h \rightarrow 0$.

Proof. Subject to the quasi-uniformity condition on the sequence of partitions, we have

$$
\|b\|_{\mathcal{T}_{h}, \Gamma}=\left(\sum_{s \in \mathcal{S}_{h}} h_{s}^{-1}\|b\|_{s}^{2}\right)^{1 / 2} \leq 2 \underline{C}^{-1 / 2} h^{-1 / 2}\left(\sum_{s \in \mathcal{S}_{h}}\|b\|_{s}^{2}\right)^{1 / 2}=2 \underline{C}^{-1 / 2} h^{-1 / 2}\|b\|_{\Gamma}
$$

It then follows from (47) and (48) that

$$
\begin{aligned}
\left\|u-u_{h}\right\|_{1, \Omega \backslash \Gamma} \leq & \left(1+C_{P} \underline{c}_{A}^{-1} \bar{c}_{A}\right) \inf _{v_{h} \in \boldsymbol{V}_{h}}\left\|u-v_{h}\right\|_{1, \Omega \backslash \Gamma} \\
& +2^{-1 / 2} C_{P} \underline{c}_{A}^{-1} \bar{c}_{A} C_{\Gamma} M^{1 / 2}\|b\|_{\mathcal{T}_{h}, \Gamma} \\
\leq & \mathscr{C}+\mathscr{C} h^{-1 / 2}
\end{aligned}
$$

and the assertion follows in the limit $h \rightarrow 0$.

The potential divergence of the bound in Corollary 3 does of course not immediately imply that the error in the WSM approximation itself increases as $h \rightarrow 0$. However, Theorem 4 below asserts that $\left\|u-u_{h}\right\|_{\Omega}$ vanishes as $h \rightarrow 0$. From this result, the continuity of $u_{h}$ and the discontinuity of $u$, one can infer that, indeed, $\left\|u-u_{h}\right\|_{1, \Omega \backslash \Gamma}$ must diverge as $h \rightarrow 0$.

Theorem 2 conveys that for each $h>0$, it holds that $u-u_{h} \in L^{2}(\Omega)$. Based on the AubinNitsche Lemma, we can can therefore construct an estimate of the error in the WSM approximation in the $\boldsymbol{L}^{2}$-norm. The estimate is formulated in Theorem 4 below. Some auxiliary conditions on the domain $\Omega$ are required, as specified in the premises of the theorem.

Theorem 4 (Global approximation properties of WSM in the $\boldsymbol{L}^{2}$-norm). Assume that the conditions of Lemma 1 hold. In addition, assume that $\Omega$ is convex or of class $C^{2}$. Let $u$ denote the solution to (11) and let $u_{h} \in \boldsymbol{V}_{h}$ denote the WSM approximation according to (23). Let $\boldsymbol{T}_{h}$ denote the intersection of $\gamma_{\Gamma}\left(\boldsymbol{V}_{h}\right)$ with $\boldsymbol{H}_{0}^{1 / 2}(\Gamma)$. It holds that

$$
\begin{aligned}
\left|\left(u-u_{h}, \varphi\right)\right| \leq & \mathscr{C}\left(\left|u-u_{h}\right|_{1, \Omega \backslash \Gamma}+\|b\|_{\mathcal{T}_{h}, \Gamma}\right) \inf _{v_{h} \in \boldsymbol{V}_{h}}\left|z_{\varphi}-v_{h}\right|_{1, \Omega} \\
+ & \mathscr{C} \inf _{\lambda_{h} \in \boldsymbol{T}_{h}}\left(\|\varphi\|_{\Omega}\left\|b-\lambda_{h}\right\|_{\Gamma}+\cdots\right. \\
& \left.\quad\left(\left\|\lambda_{h}\right\|_{1 / 2, \Gamma}+\left\|\lambda_{h}\right\|_{\mathcal{T}_{h}, \Gamma}\right) \inf _{w_{h} \in \boldsymbol{V}_{h}}\left|z_{\varphi}-w_{h}\right|_{1, \Omega \backslash \Gamma}\right)
\end{aligned}
$$

for arbitrary $\varphi \in \boldsymbol{L}^{2}(\Omega)$ and a corresponding $z_{\varphi} \in \boldsymbol{H}^{2}(\Omega) \cap \boldsymbol{H}_{0, \mathcal{D}}^{1}(\Omega)$ such that $\left\|z_{\varphi}\right\|_{2, \Omega} \leq \mathscr{C}\|\varphi\|_{\Omega}$, with $\|\cdot\|_{\mathcal{T}_{h}, \Gamma}$ defined by (43). 
Proof. For arbitrary $\varphi \in \boldsymbol{L}^{2}(\Omega)$, consider the dual problem (in the sense of distributions):

$$
\begin{array}{rlrl}
-\operatorname{div} \sigma\left(z_{\varphi}\right) & =\varphi & & \text { in } \Omega \\
z_{\varphi}=0 & & \text { on } \mathcal{D} \\
\sigma_{n}\left(z_{\varphi}\right) & =0 & & \text { on } \mathcal{N}
\end{array}
$$

or, equivalently, in weak form:

$$
z \in \boldsymbol{H}_{0, \mathcal{D}}^{1}(\Omega): \quad a_{\Gamma}\left(v, z_{\varphi}\right)=(v, \varphi)_{\Omega} \quad \forall v \in \boldsymbol{H}_{0, \mathcal{D}}^{1}(\Omega)
$$

By virtue of the smoothness conditions on the elasticity tensor (27), the conditions on the domain, and the smoothness of the (homogeneous) boundary data in (55b) and (55c), the dual problem (55) possesses an elliptic-regularity property (see, e.g., [8, 1]). The regularity property implies that the dual solution resides in $\boldsymbol{H}^{2}(\Omega) \cap \boldsymbol{H}_{0, \mathcal{D}}^{1}(\Omega)$ and satisfies the estimate $\left\|z_{\varphi}\right\|_{2, \Omega} \leq \mathscr{C}\|\varphi\|_{\Omega}$.

Denoting by $\ell_{b} \in \boldsymbol{H}_{0, \mathcal{D}}^{1}(\Omega \backslash \Gamma)$ a suitable lift of $b$ such that $\left[\left[\ell_{b}\right]\right]=b$, it holds that $u-\ell_{b} \in$ $\boldsymbol{H}_{0, \mathcal{D}}^{1}(\Omega)$. Moreover, the WSM approximation $u_{h}$ resides in $\boldsymbol{H}_{0, \mathcal{D}}^{1}(\Omega)$. The dual problem 56 therefore gives:

$$
\begin{aligned}
\left(u-u_{h}, \varphi\right)_{\Omega} & =\left(u-\ell_{b}, \varphi\right)_{\Omega}+\left(\ell_{b}, \varphi\right)_{\Omega}-\left(u_{h}, \varphi\right)_{\Omega} \\
& =a_{\Gamma}\left(u-\ell_{b}, z_{\varphi}\right)+\left(\ell_{b}, \varphi\right)_{\Omega}-a_{\Gamma}\left(u_{h}, z_{\varphi}\right) \\
& =a_{\Gamma}\left(u, z_{\varphi}\right)-a_{\Gamma}\left(u_{h}, z_{\varphi}\right)-\left(a_{\Gamma}\left(\ell_{b}, z_{\varphi}\right)-\left(\ell_{b}, \varphi\right)_{\Omega}\right)
\end{aligned}
$$

By means of (33), the term in parenthesis in the ultimate expression can be identified as the weak formulation of $\left(b,\left\{\sigma_{\nu}\left(z_{\varphi}\right)\right\}\right)_{\Gamma}$. However, for $z_{\varphi} \in \boldsymbol{H}^{2}(\Omega) \cap \boldsymbol{H}_{0, \mathcal{D}}^{1}(\Omega)$, the trace theorem asserts that $\sigma_{\nu}\left(z_{\varphi}\right) \in \boldsymbol{L}^{2}(\Gamma)$ and $\left(b,\left\{\sigma_{\nu}\left(z_{\varphi}\right)\right\}\right)_{\Gamma}$ coincides with its extension to a duality pairing. From the weak form of the Volterra dislocation problem (11) it moreover follows that $a_{\Gamma}\left(u, z_{\varphi}\right)=l\left(z_{\varphi}\right)$. The Galerkin-orthogonality property of the WSM approximation 23 then yields the following identities:

$$
\begin{aligned}
\left(u-u_{h}, \varphi\right)_{\Omega} & =l\left(z_{\varphi}-v_{h}\right)-a_{\Gamma}\left(u_{h}, z_{\varphi}-v_{h}\right)+\left(b,\left\{\sigma_{\nu}\left(v_{h}\right)\right\}\right)_{\Gamma}-\left(b,\left\{\sigma_{\nu}\left(z_{\varphi}\right)\right\}\right)_{\Gamma} \\
& =a_{\Gamma}\left(u-u_{h}, z_{\varphi}-v_{h}\right)-\left(b,\left\{\sigma_{\nu}\left(z_{\varphi}\right)\right\}-\left\{\sigma_{\nu}\left(v_{h}\right)\right\}\right)_{\Gamma}
\end{aligned}
$$

which are valid for all $v_{h} \in \boldsymbol{V}_{h}$.

For the first term in the final expression in (58) we can construct an appropriate bound without further digression. However, the second term, pertaining to the difference between the average traction of $z \in \boldsymbol{H}^{2}(\Omega) \cap \boldsymbol{H}_{0, \mathcal{D}}^{1}(\Omega)$ and the average of the direct evaluation of the traction of the finite-element function $v_{h} \in \boldsymbol{V}_{h}$, is more difficult to estimate. The essential complication in constructing an estimate, is that the direct evaluation of the traction of the Galerkin finite-element approximation of (56) in $\boldsymbol{V}_{h}$ does not coincide with the weak formulation, because $\boldsymbol{V}_{h} \notin \boldsymbol{H}_{\text {div } \sigma}^{1}(\Omega)$; see also [20]. Approximation results are available for the weak formulation of the traction (see, for instance, 3]) but, to our knowledge, not for the direct formulation.

To estimate the second term in (58), we will use an auxiliary Nitsche-type approximation [15] to the dual problem. We consider the broken approximation spaces $\hat{\boldsymbol{V}}_{h}$ according to $(19)$. Moreover, we define $\hat{\boldsymbol{V}}(h):=\left(\boldsymbol{H}^{2}(\Omega \backslash \Gamma) \cap \boldsymbol{H}_{0, \mathcal{D}}^{1}(\Omega \backslash \Gamma)\right)+\hat{\boldsymbol{V}}_{h}$. We equip $\hat{\boldsymbol{V}}(h)$ with the mesh-dependent inner product

$$
(u, v)_{\hat{\boldsymbol{V}}(h)}=a_{\Gamma}(u, v)+\zeta \sum_{s \in \mathcal{S}_{h}} h_{s}^{-1}([[u]],[[v]])_{s}
$$


and the corresponding norm $\|\cdot\|_{\hat{\boldsymbol{V}}(h)}$. We note that the embedding of $\boldsymbol{H}^{2}(\Omega \backslash \Gamma)$ into $\hat{\boldsymbol{V}}(h)$ is continuous, i.e., $\|v\|_{\hat{\boldsymbol{V}}(h)} \leq \mathscr{C}\|v\|_{2, \Omega \backslash \Gamma}$ for all $v \in \boldsymbol{H}^{2}(\Omega \backslash \Gamma)$. We define the bilinear operator $\hat{a}_{\Gamma}: \boldsymbol{V}(h) \times \boldsymbol{V}_{h} \rightarrow \mathbb{R}$ according to:

$$
\hat{a}_{\Gamma}(u, v)=a_{\Gamma}(u, v)-\left([[v]],\left\{\sigma_{\nu}(u)\right\}\right)_{\Gamma}+\theta \sum_{s \in \mathcal{S}_{h}}([[u]],[[v]])_{s}
$$

with $\theta$ a suitable constant. For a suitable choice of the constants $\zeta$ and $\theta$, the bilinear form in the left member of 61 is continuous and coercive on $\hat{\boldsymbol{V}}_{h} \times \hat{\boldsymbol{V}}_{h}$ and $\hat{a}_{\Gamma}(u, \cdot): \hat{\boldsymbol{V}}_{h} \rightarrow \mathbb{R}$ represents a continuous linear functional for all $u \in \boldsymbol{V}(h)$. Let $z_{\varphi_{h}} \in \hat{\boldsymbol{V}}_{h}$ denote the solution to the following Nitsche-type projection problem:

$$
z_{\varphi_{h}} \in \hat{\boldsymbol{V}}_{h}: \quad \hat{a}_{\Gamma}\left(z_{\varphi_{h}}, w_{h}\right)=\hat{a}_{\Gamma}\left(z_{\varphi}, w_{h}\right) \quad \forall w_{h} \in \hat{\boldsymbol{V}}_{h}
$$

By virtue of the continuity and coercivity of $\hat{a}_{\Gamma}$ on $\hat{\boldsymbol{V}}_{h} \times \hat{\boldsymbol{V}}_{h}$ and the continuity of $\hat{a}_{\Gamma}\left(z_{\varphi}, \cdot\right)$ on $\hat{\boldsymbol{V}}_{h}$, the projection problem 61 defines a unique element $z_{\varphi_{h}} \in \hat{\boldsymbol{V}}_{h}$ which satisfies

$$
\left\|z_{\varphi}-z_{\varphi_{h}}\right\|_{\hat{\boldsymbol{V}}(h)} \leq \mathscr{C} \inf _{w_{h} \in \hat{\boldsymbol{V}}_{h}}\left\|z_{\varphi}-w_{h}\right\|_{\hat{\boldsymbol{V}}(h)} \leq \mathscr{C} \inf _{w_{h} \in \boldsymbol{V}_{h}}\left|z_{\varphi}-w_{h}\right|_{1, \Omega}
$$

The first inequality in 62 is a straightforward consequence of Céa's lemma. The second inequality follows from $\boldsymbol{V}_{h} \subset \hat{\boldsymbol{V}}_{h}$, the continuity of functions in $\boldsymbol{V}_{h}$ and 14a.

By adding a suitable partition of zero to the second term in (58) and applying the triangle inequality, we obtain:

$$
\left|\left(b,\left\{\sigma_{\nu}\left(z_{\varphi}\right)\right\}-\left\{\sigma_{\nu}\left(v_{h}\right)\right\}\right)_{\Gamma}\right| \leq\left|\left(b,\left\{\sigma_{\nu}\left(z_{\varphi}-z_{\varphi_{h}}\right)\right\}\right)_{\Gamma}\right|+\left|\left(b,\left\{\sigma_{\nu}\left(v_{h}-z_{\varphi_{h}}\right)\right\}\right)_{\Gamma}\right|
$$

For the first term in the right-member of 63), we derive from 61] and 62):

$$
\begin{aligned}
\left|\left(b,\left\{\sigma_{\nu}\left(z_{\varphi}-z_{\varphi_{h}}\right)\right\}\right)_{\Gamma}\right|= & \mid\left(b-\left[\left[w_{h}\right]\right],\left\{\sigma_{\nu}\left(z_{\varphi}-z_{\varphi_{h}}\right)\right\}\right)_{\Gamma}+a_{\Gamma}\left(w_{h}, z_{\varphi}-z_{\varphi_{h}}\right) \cdots \\
& -\theta \sum_{s \in \mathcal{S}_{h}} h_{s}^{-1}\left(\left[\left[w_{h}\right]\right],\left[\left[z_{\varphi_{h}}\right]\right]\right)_{s} \mid \\
\leq & \left\|b-\left[\left[w_{h}\right]\right]\right\|_{\Gamma}\left(\left\|\left\{\sigma_{\nu}\left(z_{\varphi}\right)\right\}\right\|_{\Gamma}+\left\|\left\{\sigma_{\nu}\left(z_{\varphi_{h}}\right)\right\}\right\|_{\Gamma}\right) \\
& +\bar{c}_{A}\left|w_{h}\right|_{1, \Omega \backslash \Gamma}\left|z_{\varphi}-z_{\varphi_{h}}\right|_{1, \Omega \backslash \Gamma} \\
& +\theta\left\|\left[\left[w_{h}\right]\right]\right\|_{\mathcal{T}_{h}, \Gamma}\left\|\left[\left[z_{\varphi_{h}}\right]\right]\right\|_{\mathcal{T}_{h}, \Gamma}
\end{aligned}
$$

for all $w_{h} \in \hat{\boldsymbol{V}}_{h}$. The trace theorem implies $\left\|\left\{\sigma_{\nu}\left(z_{\varphi}\right)\right\}\right\|_{\Gamma} \leq \mathscr{C}\left\|z_{\varphi}\right\|_{2, \Omega} \leq \mathscr{C}\|\varphi\|_{\Omega}$. The continuity of $\hat{a}_{\Gamma}$ and $a_{\Gamma}$ implies that $\left\|\left\{\sigma_{\nu}\left(z_{\varphi_{h}}\right)\right\}\right\|_{\Gamma} \leq \mathscr{C}\left\|z_{\varphi_{h}}\right\|_{\hat{\boldsymbol{V}}(h)}$. Moreover, by virtue of 62 and the continuity of the embedding of $\boldsymbol{H}^{2}(\Omega)$ into $\hat{\boldsymbol{V}}(h)$ it holds that $\left\|z_{\varphi_{h}}\right\|_{\hat{\boldsymbol{V}}(h)} \leq \mathscr{C}\left\|z_{\varphi}\right\|_{\hat{\boldsymbol{V}}(h)} \leq \mathscr{C}\left\|z_{\varphi}\right\|_{2, \Omega} \leq \mathscr{C}\|\varphi\|_{\Omega}$. Hence, we have $\left\|\left\{\sigma_{\nu}\left(z_{\varphi}\right)\right\}\right\|_{\Gamma}+\left\|\left\{\sigma_{\nu}\left(z_{\varphi_{h}}\right)\right\}\right\|_{\Gamma} \leq \mathscr{C}\|\varphi\|_{\Omega}$. Noting that $\boldsymbol{T}_{h}=\gamma_{\Gamma}\left(\boldsymbol{V}_{h}\right) \cap \boldsymbol{H}_{0}^{1 / 2}(\Gamma)$ coincides with $\left\{\left[\left[w_{h}\right]\right]: w_{h} \in \hat{\boldsymbol{V}}_{h}\right\}$, for all $\lambda_{h} \in \boldsymbol{T}_{h}$ there exists a $w_{h} \in \hat{\boldsymbol{V}}_{h}$ such that $\left[\left[w_{h}\right]\right]=\lambda_{h}$ and $\left|w_{h}\right|_{1, \Omega \backslash \Gamma} \leq \mathscr{C}\|\lambda\|_{1 / 2, \Gamma}$. From (62)-64) we deduce:

$$
\begin{aligned}
\left|\left(b,\left\{\sigma_{\nu}\left(z_{\varphi}-z_{\varphi_{h}}\right)\right\}\right)_{\Gamma}\right| \leq & \mathscr{C}\left(\|\varphi\|_{\Omega}\left\|b-\lambda_{h}\right\|_{\Gamma}+\cdots\right. \\
& \left.\left(\left\|\lambda_{h}\right\|_{1 / 2, \Gamma}+\left\|\lambda_{h}\right\|_{\mathcal{T}_{h}, \Gamma}\right) \inf _{w_{h} \in \boldsymbol{V}_{h}}\left|z_{\varphi}-w_{h}\right|_{1, \Omega \backslash \Gamma}\right)
\end{aligned}
$$


for all $\lambda_{h} \in \boldsymbol{T}_{h}$, with $\|\cdot\|_{\mathcal{T}_{h}, \Gamma}$ according to (43). For the second term in the right-member of (63) we deduce from (47), the triangle inequality and (62):

$$
\begin{aligned}
\left|\left(b,\left\{\sigma_{\nu}\left(v_{h}-z_{\varphi_{h}}\right)\right\}\right)_{\Gamma}\right| & \leq 2^{-1 / 2} \bar{c}_{A} C_{\Gamma} M^{1 / 2}\|b\|_{\mathcal{T}_{h}, \Gamma}\left|v_{h}-z_{\varphi_{h}}\right|_{1, \Omega \backslash \Gamma} \\
& \leq \mathscr{C}\|b\|_{\mathcal{T}_{h}, \Gamma}\left(\left|z_{\varphi}-v_{h}\right|_{1, \Omega}+\inf _{w_{h} \in \boldsymbol{V}_{h}}\left|z_{\varphi}-w_{h}\right|_{1, \Omega}\right)
\end{aligned}
$$

with $\mathscr{C}$ independent of $h$. Collecting the results in (58)-(65) and taking the infimum with respect to $v_{h}$ and $\lambda_{h}$, one obtains the estimate in (54).

Under slightly stronger conditions on the regularity of the slip distribution $b$, we can derive from Theorem 4 a straightforward characterization of the asymptotic approximation properties of WSM in the $\boldsymbol{L}^{2}$-norm for quasi-uniform meshes in the limit as $h \rightarrow 0$ :

Corollary 5. Assume that the conditions of Corollary 3 and Theorem 4 hold and, moreover, $b \in$ $\boldsymbol{H}^{1}(\Gamma) \cap \boldsymbol{H}_{0}^{1 / 2}(\Gamma)$. Let $u$ denote the solution to 11) and let $u_{h} \in \boldsymbol{V}_{h}$ denote the WSM approximation according to (23). It holds that

$$
\left\|u-u_{h}\right\|_{\Omega} \leq \mathscr{C} h^{1 / 2}
$$

as $h \rightarrow 0$.

Proof. Based on the estimate $\left\|z_{\varphi}\right\|_{2, \Omega} \leq \mathscr{C}\|\varphi\|_{\Omega}$, it follows from standard interpolation theory in Sobolev spaces (see, for instance, [7, 2]) that $\inf _{v_{h} \in \boldsymbol{V}_{h}}\left|z-v_{h}\right|_{1, \Omega} \leq \mathscr{C} h\|\varphi\|_{\Omega}$. Similarly, for $b$ in $\boldsymbol{H}^{1}(\Gamma) \cap \boldsymbol{H}_{0}^{1 / 2}(\Gamma)$, we have $\inf _{\lambda_{h} \in \boldsymbol{T}_{h}}\left\|b-\lambda_{h}\right\|_{\Gamma} \leq \mathscr{C} h\|b\|_{1, \Gamma}$. Setting $\lambda_{h}^{*}=\arg \inf _{\lambda_{h} \in \boldsymbol{T}_{h}}\left\|b-\lambda_{h}\right\|_{\Gamma}$, it holds that $\left\|\lambda_{h}^{*}\right\|_{1 / 2, \Gamma} \leq \mathscr{C}$ and $\left\|\lambda_{h}^{*}\right\|_{\mathcal{T}_{h}, \Gamma} \leq \mathscr{C} h^{-1 / 2}$. From 52 we obtain $\|b\|_{\mathcal{T}_{h}, \Gamma} \leq \mathscr{C} h^{-1 / 2}$ and, in turn, it follows from Theorem 2 that $\left|u-u_{h}\right|_{1, \Omega} \leq \mathscr{C} h^{-1 / 2}$ as $h \rightarrow 0$. Inserting the above estimates into [54), we derive:

$$
\begin{aligned}
\left\|u-u_{h}\right\|_{\Omega} & =\sup _{\varphi \in \boldsymbol{L}^{2}(\Omega) \backslash\{0\}} \frac{\left|\left(u-u_{h}, \varphi\right)_{\Omega}\right|}{\|\varphi\|_{\Omega}} \\
& \leq \sup _{\varphi \in \boldsymbol{L}^{2}(\Omega) \backslash\{0\}} \frac{1}{\|\varphi\|_{\Omega}}\left(\mathscr{C} h^{-1 / 2} h\|\varphi\|_{\Omega}+\mathscr{C} h\|\varphi\|_{\Omega}+\left(1+h^{-1 / 2}\right) h\|\varphi\|_{\Omega}\right)
\end{aligned}
$$

as $h \rightarrow 0$. The estimate in $(66)$ then follows straightforwardly by combining terms.

Remark. The reinforced regularity condition $b \in \boldsymbol{H}^{1}(\Gamma) \cap \boldsymbol{H}_{0}^{1 / 2}(\Gamma)$ on the slip distribution ensures that $\inf _{\lambda_{h} \in \boldsymbol{T}_{h}}\left\|b-\lambda_{h}\right\|_{\Gamma} \leq \mathscr{C} h$ as $h \rightarrow 0$. Noting that the weaker estimate $\inf _{\lambda_{h} \in \boldsymbol{T}_{h}}\left\|b-\lambda_{h}\right\|_{\Gamma} \leq$ $\mathscr{C} h^{1 / 2}$ suffices to obtain the result in Corollary 5 , one is lead to question whether the reinforced regularity condition on $b$ is actually necessary. If the condition is dismissed, however, an interpolation estimate in the fractional Sobolev space $\boldsymbol{H}_{0}^{1 / 2}(\Gamma)$ is required. Interpolation estimates in fractional Sobolev spaces are technical (see, for instance, [6]) and the particular result required here is to our knowledge not available.

It is noteworthy that the asymptotic convergence behavior according to Corollary 5 is consistent with the notion that the continuous WSM approximation incurs an $O(1)$ error, pointwise, in the $O(h)$ neighborhood of the discontinuity composed of the intersected elements as $h \rightarrow 0$. In particular, denoting by $\Gamma_{h}$ the union of the elements for which the intersection of the fault with the closure of the element is non-empty, it holds that

$$
\|1\|_{\Gamma_{h}}=\left(\operatorname{meas}_{N}\left(\Gamma_{h}\right)\right)^{1 / 2}=O\left(h^{1 / 2}\right)
$$

as $h \rightarrow 0$, with meas $_{N}\left(\Gamma_{h}\right)$ the $N$-Lebesgue measure of $\Gamma_{h}$. 


\subsection{Local approximation properties}

Next, we consider the local approximation properties of WSM, i.e., on $\Omega$ excluding a small neighborhood of the fault. To this end, we will relate the WSM approximation to the standard Galerkin approximation associated with a locally supported lift, outside the support of the lift.

For arbitrary $\varepsilon>0$, let $\varkappa^{\varepsilon}:=\{x \in \Omega: \operatorname{dist}(x, \varkappa)<\varepsilon\}$ denote the open $\varepsilon$-neighborhood of the dislocation fault. We consider a lift $\ell_{b} \in \boldsymbol{H}_{0, \mathcal{D}}^{1}(\Omega \backslash \Gamma)$ of $b$ with compact support in $\varkappa^{\varepsilon}$. Let $\bar{u}_{h} \in \boldsymbol{V}_{h}$ denote the Galerkin approximation of the continuous complement of the solution with respect to the jump lift $\ell_{b}$ according to (17). As a straightforward consequence of Céa's lemma, it follows that $\bar{u}_{h}+\ell_{b}$ is endowed with the quasi-optimal approximation property:

$$
\left\|u-\left(\bar{u}_{h}+\ell_{b}\right)\right\|_{1, \Omega \backslash \Gamma} \leq \mathscr{C}_{v_{h} \in \boldsymbol{V}_{h}}\left\|u-\left(v_{h}+\ell_{b}\right)\right\|_{1, \Omega \backslash \Gamma}
$$

A meaningful characterization of the local approximation properties of WSM is therefore provided by an estimate for the deviation $\left\|\bar{u}_{h}+\ell_{b}-u_{h}\right\|_{1, \Omega \backslash \varkappa^{\varepsilon}}$ between the WSM approximation $u_{h}$ and the local-lift-based approximation $\bar{u}_{h}+\ell_{b}$. Note that $\varkappa^{\varepsilon} \supset \operatorname{supp}\left(\ell_{b}\right)$ implies that $\ell_{b}$ vanishes on $\Omega \backslash \varkappa^{\varepsilon}$ and, hence, the estimate pertains to the deviation between $\bar{u}_{h} \in \boldsymbol{V}_{h}$ and $u_{h} \in \boldsymbol{V}_{h}$.

The characterization of the local approximation properties of WSM in the $\boldsymbol{H}^{1}$-norm in Theorem 6 below is based on the interpolation of a particular extension of functions in $\boldsymbol{V}_{h}$ onto $\varkappa^{\varepsilon}$. Specifically, we consider an extension corresponding to the operator $E: \boldsymbol{V}_{h} \rightarrow \boldsymbol{H}^{1}(\Omega)$ :

$$
\begin{aligned}
E\left(v_{h}\right) & =v_{h} & & \text { in } \Omega \backslash \varkappa^{\varepsilon} \\
-\operatorname{div} \sigma\left(E\left(v_{h}\right)\right) & =0 & & \text { in } \varkappa^{\varepsilon}
\end{aligned}
$$

in the sense of distributions, and its optimal approximation in the norm defined by $a_{\Gamma}(\cdot, \cdot)$ :

$$
E_{h}\left(v_{h}\right)=\underset{w_{h} \in v_{h}+\boldsymbol{V}_{h, \varkappa^{\varepsilon}}}{\arg \min } a_{\Gamma}\left(E\left(v_{h}\right)-w_{h}, E\left(v_{h}\right)-w_{h}\right)
$$

where $\boldsymbol{V}_{h, \varkappa^{\varepsilon}}:=\left\{v_{h} \in \boldsymbol{V}_{h}: \operatorname{supp}\left(v_{h}\right) \subseteq \varkappa^{\varepsilon}\right\} \neq \emptyset$ denotes the class of approximation functions of which the support is confined to $\varkappa^{\varepsilon}$. Note that $\boldsymbol{V}_{h, \varkappa^{\varepsilon}} \neq \emptyset$ implies $\varepsilon \geq \mathscr{C} h$.

Equations (70a) and (71) imply that $E\left(v_{h}\right)$ and $E_{h}\left(v_{h}\right)$ coincide with $v_{h}$ outside $\varkappa^{\varepsilon}$. Inside $\varkappa^{\varepsilon}$, the extension $E\left(v_{h}\right)$ is defined by the homogeneous elasticity problem $70 \mathrm{~b}$. From $E\left(v_{h}\right) \in \boldsymbol{H}^{1}(\Omega)$ it follows that $E\left(v_{h}\right)$ is continuous at $\partial \varkappa^{\varepsilon}$ in the trace sense, which implies that $\left.770 \mathrm{~b}\right)$ is complemented by the Dirichlet boundary condition $E\left(v_{h}\right)=v_{h}$ on $\partial \varkappa^{\varepsilon}$. By virtue of the smoothness condition on the elasticity tensor in (27), the extension operator according to $(70)$ exhibits an interior regularity property on $\varkappa^{\varepsilon}$. In particular, for all $v_{h} \in \boldsymbol{V}_{h}$ it holds that $E\left(v_{h}\right) \in \boldsymbol{H}_{\mathrm{loc}}^{2}\left(\varkappa^{\varepsilon}\right)$ (i.e., $\phi E\left(v_{h}\right) \in$ $\boldsymbol{H}^{2}\left(\varkappa^{\varepsilon}\right)$ for any $\phi \in C^{\infty}(\Omega)$ with compact support in $\left.\varkappa^{\varepsilon}\right)$ and the following estimate holds for each open subset $K \Subset \varkappa^{\varepsilon}$ :

$$
\left\|E\left(v_{h}\right)\right\|_{2, K} \leq \mathscr{C}\left\|E\left(v_{h}\right)\right\|_{\varkappa^{\varepsilon}} \quad \forall v_{h} \in \boldsymbol{V}_{h}
$$

see, for instance, [8, §6.3.1] or [13, §2.3] for further details. It is important to note that estimate 772 in conjunction with the trace theorem implies that

$$
\left\|\left\{\sigma_{\nu}\left(E\left(v_{h}\right)\right)\right\}\right\|_{\varkappa} \leq \mathscr{C}\left\|E\left(v_{h}\right)\right\|_{1, \varkappa^{\varepsilon}} \quad \forall v_{h} \in \boldsymbol{V}_{h},
$$

for some $\mathscr{C}>0$ independent of $v_{h}$, provided that there exists an open subset $K \Subset \varkappa^{\varepsilon}$ such that $\varkappa \subset K$. This provision implies that the dislocation must be properly contained in $\Omega$. The 
proof of Theorem 6 involves an estimate of the difference between the average traction of $E\left(v_{h}\right)$ and its approximation $E_{h}\left(v_{h}\right)$ according to $(71)$. In general, we can estimate $\left(b,\left\{\sigma_{\nu}\left(E\left(v_{h}\right)-E_{h}\left(v_{h}\right)\right)\right\}\right)_{\Gamma}$ in the same manner as in the proof of Theorem 4 . The bound $(73)$ in combination with the optimalapproximation property of $E_{h}\left(v_{h}\right)$ in $(71)$ however suggests that in this case a sharper estimate can be established. The derivation of such a refined estimate is intricate, however, as $\left(b,\left\{\sigma_{\nu}(\cdot)\right\}\right)_{\Gamma}$ is unbounded on $\boldsymbol{H}^{1}(\Omega)$ and the estimate involves the difference between $E\left(v_{h}\right)$ and $E_{h}\left(v_{h}\right)$, which reside in different subspaces of $\boldsymbol{H}^{1}(\Omega)$. We therefore formulate the refined estimate in the form of a conjecture:

Conjecture 1. Assume that there exist an $\varepsilon^{*}>0$ and an open subset $K \Subset \varkappa^{\varepsilon}$ such that $\varkappa \subset K$. Consider a sequence of approximation spaces $\boldsymbol{V}_{\mathcal{H}}$, the extension operator according to (70) and its approximation according to (71). For all $\varepsilon \geq \varepsilon^{*}$ and all $v_{h} \in \boldsymbol{V}_{h}$, it holds that

$$
\left\|\left\{\sigma_{\nu}\left(E\left(v_{h}\right)-E_{h}\left(v_{h}\right)\right)\right\}\right\|_{\varkappa} \leq \mathscr{C}\left\|E\left(v_{h}\right)-E_{h}\left(v_{h}\right)\right\|_{1, \varkappa^{\varepsilon}}
$$

for some constant $\mathscr{C}>0$ independent of $h$.

Theorem 6 below presents a general characterization of the local approximation properties of WSM, independent of Conjecture 1, and a refinement, which is contingent on the conjecture.

Theorem 6 (Local approximation properties of WSM in the $\boldsymbol{H}^{1}$-norm). Assume that there exist an $\varepsilon^{*}>0$ and an open subset $K \Subset \varkappa^{\varepsilon}$ such that $\varkappa \subset K$. For arbitrary $\varepsilon \geq \varepsilon^{*}$, let $\ell_{b} \in \boldsymbol{H}_{0, \mathcal{D}}^{1}(\Omega \backslash \Gamma)$ denote a lift of $b$ such that $\operatorname{supp}\left(\ell_{b}\right) \subset \varkappa^{\varepsilon}$. Given a sequence of approximation spaces $\boldsymbol{V}_{\mathcal{H}}$, for each $h \in \mathcal{H}$ let $\bar{u}_{h}$ denote the approximation of the continuous complement corresponding to $\ell_{b}$ in (17) and let $u_{h} \in \boldsymbol{V}_{h}$ denote the WSM approximation according to (23). Consider the extension operator in (70) and its approximation in $\boldsymbol{V}_{h}$ according to (71). It holds that

$$
\begin{aligned}
\left\|\bar{u}_{h}+\ell_{b}-u_{h}\right\|_{1, \Omega \backslash \varkappa^{\varepsilon}}^{2} \leq & \mathscr{C}\left\|\ell_{b}\right\|_{1, \varkappa^{\varepsilon} \backslash \Gamma}\left\|E\left(\bar{u}_{h}-u_{h}\right)-E_{h}\left(\bar{u}_{h}-u_{h}\right)\right\|_{1, \varkappa^{\varepsilon}} \\
& +\mathscr{C}\left|\left(b,\left\{\sigma_{\nu}\left(E\left(\bar{u}_{h}-u_{h}\right)-E_{h}\left(\bar{u}_{h}-u_{h}\right)\right)\right\}\right)_{\Gamma}\right|
\end{aligned}
$$

For some constant $\mathscr{C}>0$ independent of $h$ and $\varepsilon$. If, in addition, Conjecture 1 holds, then

$$
\left\|\bar{u}_{h}+\ell_{b}-u_{h}\right\|_{1, \Omega \backslash \varkappa^{\varepsilon}} \leq \mathscr{C}\left(\left\|\ell_{b}\right\|_{1, \varkappa^{\varepsilon} \backslash \Gamma}+\|b\|_{\Gamma}\right) \sup _{v_{h} \in \boldsymbol{V}_{h} w_{h} \in v_{h}+\boldsymbol{V}_{h, \varkappa^{\varepsilon}}} \frac{\left\|E\left(v_{h}\right)-w_{h}\right\|_{1, \varkappa^{\varepsilon}}}{\left\|v_{h}\right\|_{1, \Omega \backslash \varkappa^{\varepsilon}}}
$$

for some constant $\mathscr{C}>0$ independent of $h$ and $\varepsilon$.

Proof. We use the condensed notation $E_{h}:=E_{h}\left(\bar{u}_{h}-u_{h}\right)$. Noting that (71) implies that $E_{h}$ coincides with $\bar{u}_{h}-u_{h}$ on $\Omega \backslash \varkappa^{\varepsilon}$ and that $\operatorname{supp}\left(\ell_{b}\right) \subset \varkappa^{\varepsilon}$, it holds that

$$
\left\|\bar{u}_{h}+\ell_{b}-u_{h}\right\|_{1, \Omega \backslash \varkappa^{\varepsilon}}=\left\|\bar{u}_{h}-u_{h}\right\|_{1, \Omega \backslash \varkappa^{\varepsilon}} \leq\left\|E_{h}\right\|_{1, \Omega}
$$

Using the Poincaré inequality 15 and the strong positivity of $a_{\Gamma}(\cdot, \cdot)$ according to $14 \mathrm{~b}$, we obtain the following bound:

$$
\left\|E_{h}\right\|_{1, \Omega}^{2} \leq\left(1+C_{p}\right) \underline{c}_{A}^{-1}\left|a_{\Gamma}\left(E_{h}, E_{h}\right)\right|
$$

By introducing a suitable partition of zero, we derive from the WSM formulation (23) and the lift-based Galerkin approximation (17):

$$
\begin{aligned}
a_{\Gamma}\left(E_{h}, E_{h}\right) & =a_{\Gamma}\left(\bar{u}_{h}-u_{h}, E_{h}\right)+a_{\Gamma}\left(E_{h}-\left(\bar{u}_{h}-u_{h}\right), E_{h}\right) \\
& =\left(b,\left\{\sigma_{\nu}\left(E_{h}\right)\right\}\right)_{\Gamma}-a_{\Gamma}\left(\ell_{b}, E_{h}\right)+a_{\Gamma}\left(E_{h}-\left(\bar{u}_{h}-u_{h}\right), E_{h}\right)
\end{aligned}
$$


By virtue of (70b), $a_{\Gamma}\left(v_{h}, E\left(\bar{u}_{h}-u_{h}\right)\right)$ vanishes for all $v_{h} \in \boldsymbol{V}_{h, \varkappa^{\varepsilon}}$. The optimality condition associated with (71) therefore reduces to:

$$
a_{\Gamma}\left(v_{h}, E_{h}\right)=0 \quad \forall v_{h} \in \boldsymbol{V}_{h, \varkappa^{\varepsilon}} .
$$

Noting that $E_{h}-\left(\bar{u}_{h}-u_{h}\right) \in \boldsymbol{V}_{h, \varkappa^{\varepsilon}}$, Equation (80) implies that the right-most term in the ultimate expression in (79) vanishes. By virtue of the interior regularity of $E\left(\bar{u}_{h}-u_{h}\right)$, the following identity holds:

$$
a_{\Gamma}\left(\ell_{b}, E\left(\bar{u}_{h}-u_{h}\right)\right)=\left(b,\left\{\sigma_{\nu}\left(E\left(\bar{u}_{h}-u_{h}\right)\right)\right\}\right)_{\Gamma}
$$

Let us note that (81) corresponds to the (admissible) identification of the duality pairing between $b \in \boldsymbol{H}_{0}^{1 / 2}(\varkappa)$ and $\left\{\sigma_{\nu}\left(E\left(\bar{u}_{h}-u_{h}\right)\right)\right\} \in \boldsymbol{H}^{-1 / 2}(\varkappa)$ to an $\boldsymbol{L}^{2}$ inner product. From 79 -81) and the triangle inequality we then deduce that:

$$
\left|a_{\Gamma}\left(E_{h}, E_{h}\right)\right| \leq\left|a_{\Gamma}\left(\ell_{b}, E\left(\bar{u}_{h}-u_{h}\right)-E_{h}\right)\right|+\left|\left(b,\left\{\sigma_{\nu}\left(E\left(\bar{u}_{h}-u_{h}\right)-E_{h}\right)\right\}\right)_{\Gamma}\right|
$$

We recall that $E\left(\bar{u}_{h}-u_{h}\right)-E_{h}$ vanishes on $\Omega \backslash \varkappa^{\varepsilon}$. Estimate 75 then follows straightforwardly from (77), 278), 82 and the continuity of $a_{\Gamma}(\cdot, \cdot)$ according to (14a).

To prove the auxiliary assertion (76), we note that $\left\|E\left(v_{h}\right)-E_{h}\left(v_{h}\right)\right\|_{1, \Omega}$ in fact depends only on the trace of $v_{h}$ on $\partial \varkappa^{\varepsilon}$ and, by the trace theorem, it holds that $\left\|E\left(v_{h}\right)-E_{h}\left(v_{h}\right)\right\|_{1, \Omega} \leq \mathscr{C}\left\|v_{h}\right\|_{1, \Omega \backslash \varkappa^{\varepsilon}}$. Therefore, if Conjecture 1 holds, we obtain from 75 :

$$
\begin{aligned}
\left\|\bar{u}_{h}-u_{h}\right\|_{1, \Omega \backslash \varkappa^{\varepsilon}}^{2} & \leq \mathscr{C}\left(\left\|\ell_{b}\right\|_{1, \varkappa^{\varepsilon} \backslash \Gamma}+\|b\|_{\Gamma}\right)\left\|E\left(\bar{u}_{h}-u_{h}\right)-E_{h}\left(\bar{u}_{h}-u_{h}\right)\right\|_{1, \varkappa^{\varepsilon}} \\
& \leq \mathscr{C}\left(\left\|\ell_{b}\right\|_{1, \varkappa^{\varepsilon} \backslash \Gamma}+\|b\|_{\Gamma}\right) \sup _{v_{h} \in V_{h}} \frac{\left\|E\left(v_{h}\right)-E_{h}\left(v_{h}\right)\right\|_{\varkappa^{\varepsilon}}}{\left\|v_{h}\right\|_{1, \Omega \backslash \varkappa^{\varepsilon}}}\left\|\bar{u}_{h}-u_{h}\right\|_{1, \Omega \backslash \varkappa^{\varepsilon}}
\end{aligned}
$$

Estimate $(76)$ follows directly from the identity in $(77)$, the ultimate bound in (83) and the definition of $E_{h}$ in (71).

Theorem 6 essentially implies that if the lift-based approximation (17) displays optimal global convergence, then the WSM approximation 23) displays optimal local convergence.

Corollary 7. Assume that Conjecture 1 holds and that there exist an $\varepsilon^{*}>0$ and an open subset $K \Subset \varkappa^{\varepsilon^{*}}$ such that $\varkappa \subset K$. Assume that $A_{i j k l} \in C^{k+1}(\bar{\Omega})$ for some integer $k \geq 0$ and that $\Omega$ is convex or of class $C^{k+2}$. Assume that $b$ admits a sufficiently smooth local lifting, in particular, that there exists an $\ell_{b}$ such that $\left\{\sigma_{\nu}\left(\ell_{b}\right)\right\}=0$, $\operatorname{div} \sigma\left(\ell_{b}\right) \in \boldsymbol{H}^{k}(\Omega)$ and $\operatorname{supp}\left(\ell_{b}\right) \subset \varkappa^{\varepsilon}$. Let $\boldsymbol{V}_{\mathcal{H}}$ denote a sequence of $\boldsymbol{H}^{1}(\Omega)$-conforming piecewise polynomial approximation spaces of degree $p \geq 1$ with approximation property (18). Let $u$ denote the solution to the Volterra dislocation problem (11) and let $u_{\mathcal{H}}$ denote the sequence of WSM approximations (23) corresponding to the approximation spaces $\boldsymbol{V}_{\mathcal{H}}$. It holds that

$$
\left\|u-u_{h}\right\|_{1, \Omega \backslash \varkappa^{\varepsilon}} \leq \mathscr{C} h^{l}
$$

as $h \rightarrow 0$ with $\mathscr{C}>0$ independent of $h$ and $l=\min \{p, k+1\}$.

Proof. Note that the following integration-by-parts identity holds for all $v \in \boldsymbol{H}^{1}(\Omega)$ :

$$
a_{\Gamma}\left(\ell_{b}, v\right)=\int_{\partial(\Omega \backslash \Gamma)} v \cdot \sigma_{n}\left(\ell_{b}\right)-\int_{\Omega \backslash \Gamma} v \cdot \operatorname{div} \sigma\left(\ell_{b}\right)=\int_{\Gamma} v \cdot\left\{\sigma_{\nu}\left(\ell_{b}\right)\right\}-\int_{\Omega \backslash \Gamma} v \cdot \operatorname{div} \sigma\left(\ell_{b}\right)
$$


The second identity follows by rearranging terms and applying (8). The first term in the ultimate expression in 85) vanishes by virtue of the conditions on $\ell_{b}$. Moreover, because $l(v)=(f, v)=0$, the right member of 12 corresponds to

$$
l(v)-a_{\Gamma}\left(\ell_{b}, v\right)=-\int_{\Omega \backslash \Gamma} v \cdot \operatorname{div} \sigma\left(\ell_{b}\right)
$$

By virtue of $\operatorname{div} \sigma\left(\ell_{b}\right) \in \boldsymbol{H}^{k}(\Omega)$ and the conditions on the elasticity tensor and the domain, it then holds that the continuous complement $\bar{u}$ in 12 resides in $\boldsymbol{H}^{k+2}(\Omega)$; see, for instance, 8 , Theorem 6.3.5]. The quasi-optimality of the Galerkin approximation (17) in combination with the optimal approximation properties of $\boldsymbol{V}_{h}$ according to 18 implies that $\left\|\bar{u}-\bar{u}_{h}\right\|_{1, \Omega} \leq \mathscr{C} h^{l}$. Moreover, the conditions on the elasticity tensor imply that $E\left(v_{h}\right) \in \boldsymbol{H}_{\mathrm{loc}}^{k+2}\left(\varkappa^{\varepsilon}\right)$ and, in turn, 18, yields:

$$
\sup _{v_{h} \in \boldsymbol{V}_{h}} \inf _{w_{h} \in v_{h}+\boldsymbol{V}_{h, \varkappa^{\varepsilon}}} \frac{\left\|E\left(v_{h}\right)-w_{h}\right\|_{1, \varkappa^{\varepsilon}}}{\left\|v_{h}\right\|_{1, \Omega \backslash \varkappa^{\varepsilon}}} \leq \mathscr{C} h^{l}
$$

The estimate 84 then follows from

$$
\left\|u-u_{h}\right\|_{1, \Omega \backslash \varkappa^{\varepsilon}} \leq\left\|\bar{u}-\bar{u}_{h}\right\|_{1, \Omega}+\left\|\bar{u}_{h}+\ell_{b}-u_{h}\right\|_{1, \Omega \backslash \varkappa^{\varepsilon}}
$$

and Theorem 6 .

\section{Numerical results}

We will assess the approximation properties of the Weakly-enforced Slip Method on the basis of three different test cases. All three test cases are designed to have analytical results available. This allows us to compare the exact solution of boundary value problem (5) with the WSM solution (23) and study the behavior of errors and convergence under refinement of the finite finite-element mesh.

The considered test cases are:

I. a two-dimensional infinite domain loaded in plane strain, with a straight, finite dislocation and smooth slip distribution,

II. a three-dimensional semi-infinite domain with traction-free surface, a planar, non-rupturing dislocation and constant slip, and

III. a three-dimensional semi-infinite domain with traction-free surface, a planar, surface rupturing dislocation and constant slip.

All three test cases are in principle set on infinite domains. To make the problems amenable to treatment by the finite-element method, we truncate the domains, and restrict the analyses to suitably large, but finite, neighbourhoods of the dislocation. In order to focus on the treatment of the dislocations, boundary truncation errors are controlled by constraining the finite-element approximation to the analytical solution at the artificial lateral boundaries.

Let us note that of the three test cases, only the first one is by design in full accordance with the theory developed in Section 4 Test case II and III feature a piecewise constant slip to match available analytical solutions, violating the condition of Lemma 1 which states that the slip can be 


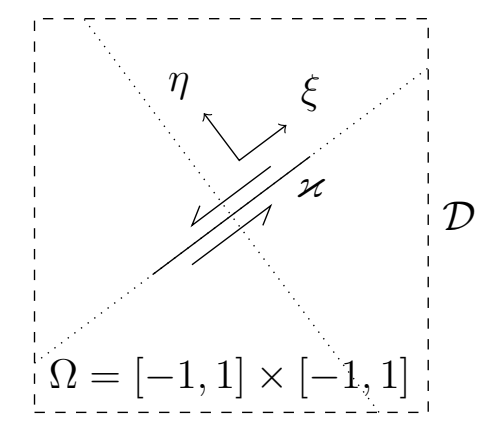

Figure 4: Schematic overview of test case I. The dashed line marks the computational domain, which is a truncation of the actual (infinite) domain. A unit-length dislocation $\varkappa$ is placed at an $\arctan (3 / 4)$ angle with the horizontal axis at the center of the computational domain.

lifted into $\boldsymbol{H}_{0, \mathcal{D}}^{1}(\Omega \backslash \Gamma)$, i.e., $b \in \boldsymbol{H}_{0}^{1 / 2}(\varkappa)$. Test case III moreover considers a rupturing fault and, accordingly, it has nonzero slip at the intersection with the domain boundary. The results below convey that the main results of the theory nonetheless uphold, suggesting that the conditions under which they apply can be relaxed.

\subsection{Test case I: $2 \mathrm{D}$ plane strain}

The first test case is a line dislocation in plane strain in an infinite two-dimensional isotropic domain. The dislocation is straight and of unit length, with a smoothly varying slip that is tangent to the dislocation line, making it a pure shear dislocation. Figure 4 shows a schematic of the computational setup.

Introducing an arclength coordinate $\xi$ along the fault, we consider a smooth, piecewise quadratic slip distribution according to:

$$
b: \mathbb{R} \rightarrow b_{0}\left\{\begin{array}{lcc}
\frac{3}{2}+6 \xi+6 \xi^{2} & -\frac{1}{2}<\xi<-\frac{1}{6} \\
1-12 \xi^{2} & -\frac{1}{6}<\xi< & \frac{1}{6} \\
\frac{3}{2}-6 \xi+6 \xi^{2} & \frac{1}{6}<\xi< & \frac{1}{2} \\
0 & \text { otherwise }
\end{array}\right.
$$

The dislocation, which corresponds to the support of the slip, is located in the interval $\xi \in\left[-\frac{1}{2}, \frac{1}{2}\right]$. The slip is symmetric, with zero displacement at the tips and smoothly opening and closing. We set the scaling factor $b_{0}=0.1$. The value of $b_{0}$ is however non-essential on account of the linearity of the problem. An analytical solution to this problem can be constructed for an infinite, homogeneous, isotropic domain, by adapting the well known solution for edge dislocations [10. Introducing a perpendicular coordinate $\eta$, the resulting displacement along the $(\xi, \eta)$ coordinates is expressed in terms of Lamé parameters $\lambda$ and $\mu$ as

$$
u(\xi, \eta)=b_{0}\left[6 U\left(\xi-\frac{1}{2}, \eta\right)-18 U\left(\xi-\frac{1}{6}, \eta\right)+18 U\left(\xi+\frac{1}{6}, \eta\right)-6 U\left(\xi+\frac{1}{2}, \eta\right)\right]
$$

where

$$
U(\xi, \eta)=\left(\begin{array}{c}
2 \frac{2 \lambda+3 \mu}{\lambda+2 \mu} \xi \eta \\
-\frac{\mu}{\lambda+2 \mu} \xi^{2}-\frac{2 \lambda+\mu}{\lambda+2 \mu} \eta^{2}
\end{array}\right) \log \sqrt{\xi^{2}+\eta^{2}}+\left(\begin{array}{c}
\frac{3 \lambda+4 \mu}{\lambda+2 \mu} \eta^{2}-\xi^{2} \\
2 \frac{\lambda}{\lambda+2 \mu} \xi \mu
\end{array}\right) \arctan \frac{\xi}{\mu}
$$




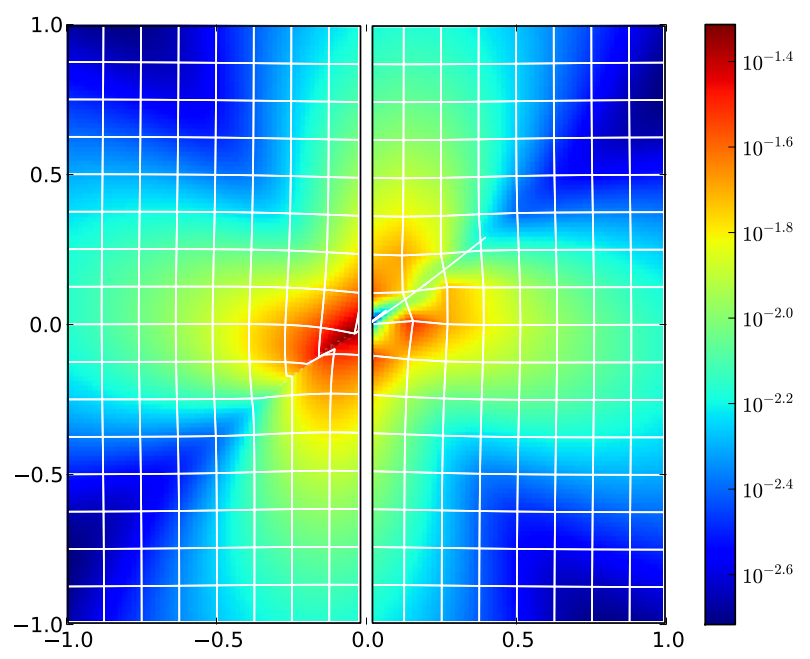

Figure 5: Side by side view of the discontinuous exact solution of test case I (left) with the continuous WSM approximation on a $16 \times 16$ mesh and linear shape functions (right). Color represents displacement magnitude on a log scale. Mesh lines on the left are introduced to indicate displacements and to facilitate comparison.

A finite sized problem is obtained by truncating the domain to $\Omega=(-1,1)^{2}$ and introducing a Dirichlet condition at the boundary $\partial \Omega=\mathcal{D}$ with data corresponding to the exact solution. The fault is located in the center of the domain at an angle of $\arctan (3 / 4)$. All numerical results are generated for Lamé parameters $\lambda=1, \mu=1$.

Figure 5 shows the exact solution of Eqn. (90) (left) in the deformed configuration, side by side with the WSM approximation for linear shape functions on a $16 \times 16$ mesh (right). The colors indicate displacement magnitude on a $\log$ scale. The grid-line pattern represents the distortion of a mesh that is uniform in the undeformed configuration. The lines coincide with element edges for the finite element computation on the right, and are matched on the left to facilitate visual comparison. Because the test case is symmetric, we expect the images to be approximately rotationally symmetric. We observe that the rotational symmetry breaks at the dislocation, where the exact solution is discontinuous, while the WSM approximation is continuous throughout the domain.

The global symmetry of the displacement pattern of Figure 5 indicates that the error induced by the WSM approximation diminish with distance from the dislocation. To quantify this observation, Figure 6 shows the errors for a $16 \times 16$ mesh (left), and for a sequence of meshes along the line B-B' perpendicular to the fault (right). The latter shows that for every mesh the error decays exponentially with increasing distance from the fault, with the exception of a narrow zone in the vicinity of the dislocation, where the error displays a transition to the constant error $\frac{1}{2} b_{0}$ at the dislocation. One may observe that the error at the dislocation is independent of element size. Away from the dislocation, errors are seen to decrease with element size, approximately reducing by $10^{0.6} \approx 4$ whenever the mesh width is halved. This error reduction provides a first indication 

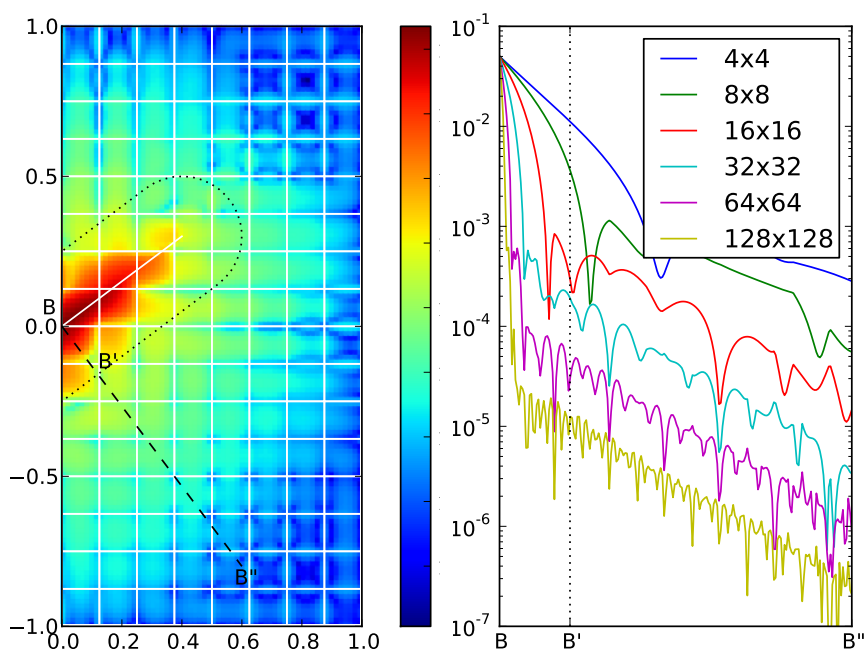

Figure 6: Absolute value of the displacement error for the $16 \times 16$ mesh result of Figure 5 (left), side by side with error evaluations along the line B-B" for a sequence of meshes of increasing density. The errors shown on the left thus correspond with the 3rd (red) curve on the right.

that local $\boldsymbol{L}^{2}$ convergence is optimal at $O\left(h^{p+1}\right)$ as $h \rightarrow 0$.

Convergence of the WSM approximation under mesh refinement is further examined in Figure 7 . which displays the $\boldsymbol{L}^{2}$-norm and $\boldsymbol{H}^{1}$-norm of the error for both linear $(p=1)$ and quadratic $(p=2)$ shape functions. The curve marked 'global' shows the error integrated over the entire computational domain. The curve marked 'local' shows the error integrated over the domain excluding an 0.1neighborhood of the dislocation, corresponding to the dotted area in Figure6. We observe that for both linear and quadratic approximations, the global $\boldsymbol{H}^{1}$-norm of the error diverges as $O\left(h^{-1 / 2}\right)$ as $h \rightarrow 0$, while the global $\boldsymbol{L}^{2}$-norm of the error converges as $O\left(h^{-1 / 2}\right)$, independent of the order of approximation. This asymptotic behavior is in accordance with the estimates in Corollaries 3 and 5. Figure 7 moreover corroborates that the local $\boldsymbol{H}^{1}$-norm of the error converges as $O\left(h^{p}\right)$ as $h \rightarrow 0$, in agreement with the estimate in Corollary 7. The local $\boldsymbol{L}^{2}$-norm of the error, for which no theory was developed, also displays an optimal convergence rate of $O\left(h^{p+1}\right)$ as $h \rightarrow 0$.

\subsection{Test case II: 3D traction-free halfspace}

The second test case is a planar dislocation buried in a semi-infinite, three dimensional, homogeneous, isotropic domain with a flat, traction-free surface. The dislocation is a rectangular plane, the sides of which are displaced over a constant distance in both strike and in dip direction, such that in geodetic terms the setting is that of an oblique left-lateral thrust fault. Figure 8 shows a schematic of the computational setup. Analytical solutions to this problem have been derived by Okada [16, 17]. We use homogeneous Lamé constants $\lambda=1, \mu=1$ for all subsequent computations.

Computations are performed on a truncated domain $\Omega=[-1,1] \times[-1,1] \times[-1,0]$, with Dirichlet conditions enforcing the Okada solution at the five truncation planes. Figure 9 shows displacements along the intersection planes A-A' and B-B' indicated in Figure 8. The topmost figure shows the exact solution according to Okada's equations. The middle and bottom figures display the WSM 

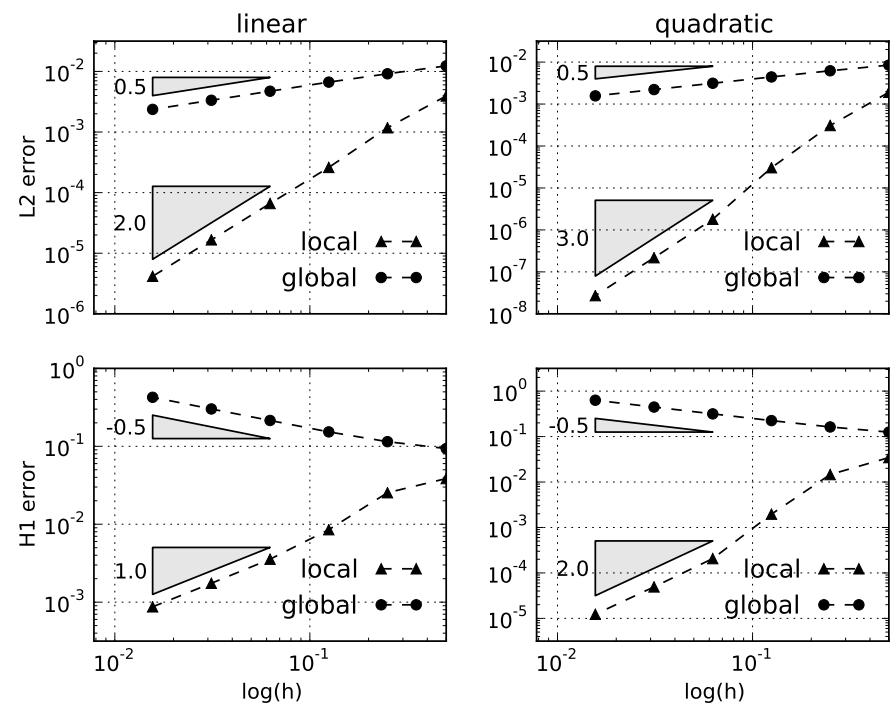

Figure 7: Mesh convergence of WSM applied to test case I, showing the $\boldsymbol{L}^{2}$-norm (top) and $\boldsymbol{H}^{1}$-norm (bottom) of the error for linear (left) and quadratic (right) shape functions. The markers corresponds to mesh sequences of $\{4 \times 4,8 \times 8, \ldots, 128 \times 128\}$ elements are considered. The global error is computed by integration over the entire computational domain $\Omega$, the local error by integration over the $>0.1$ distance exterior around $\varkappa$, bounded by the dotted line in Figure 6

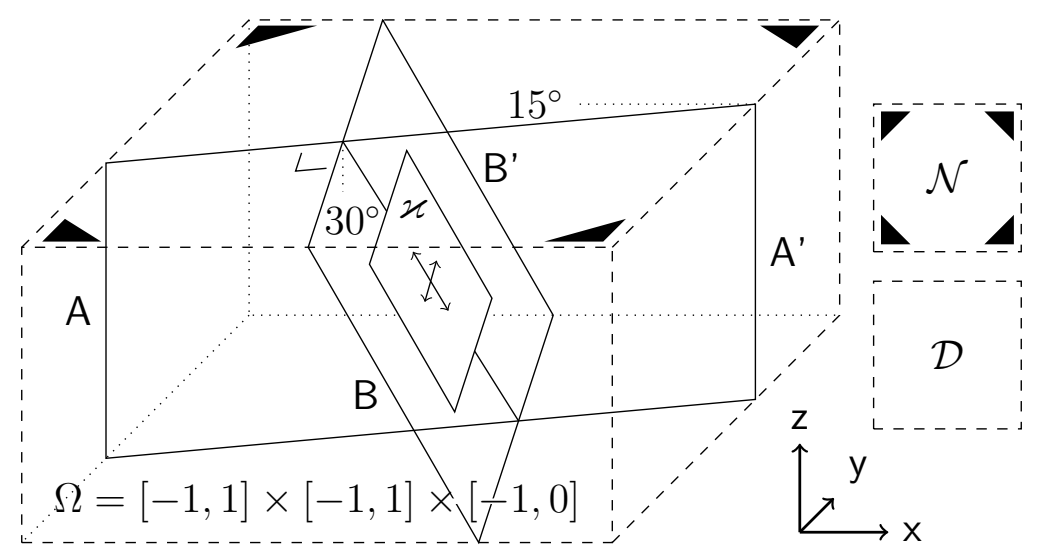

Figure 8: Schematic overview of test case II. A $1 \times 3^{-1 / 2}$ sized dislocation plane is positioned at $15^{\circ}$ strike and $30^{\circ}$ dip, spanning a $0.25-0.75$ depth range. The surface is traction free. Dashed faces mark the computational domain, which is a truncation of the actual (semi-infinite) domain. The fault is represented by the B-B' plane, where at $\varkappa$ the medium is dislocated by a constant 0.2 displacement in strike direction and a constant 0.1 displacement in dip direction. The perpendicular intersection plane $\mathrm{A}-\mathrm{A}^{\prime}$ serves visualization purposes only; see Figures 9 and 12 

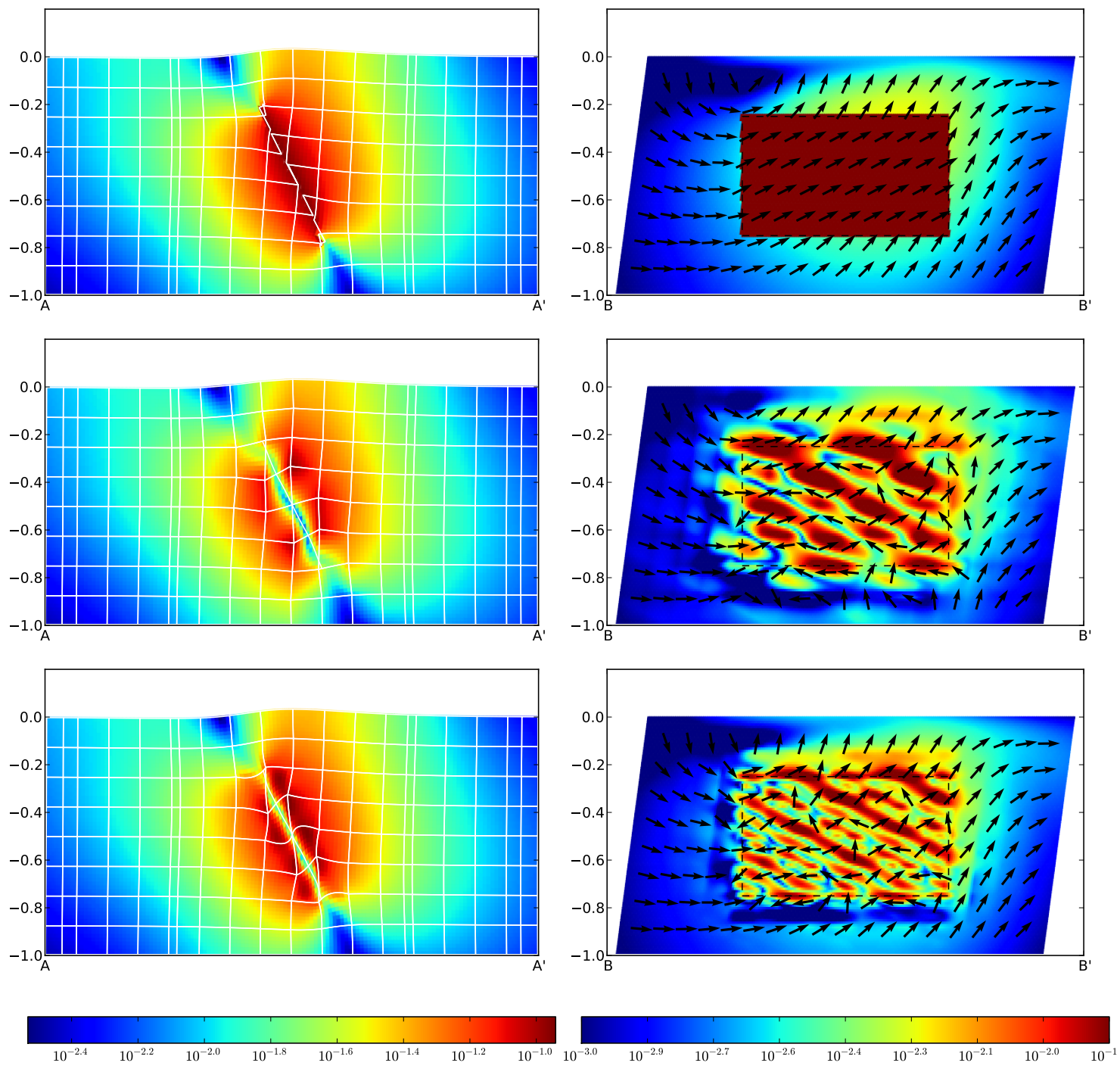

Figure 9: Cross-section views of the displacement field of test case II, corresponding to the A-A' and B-B' intersection planes of Figure 8, exact displacement as derived by Okada 17] (top) and WSM approximation on a $16 \times 16 \times 8$ mesh with linear (middle) and quadratic shape functions (bottom). Colors indicate the displacement magnitude on a logarithmic scale analogous to Figure 5 Arrows indicate direction only. 

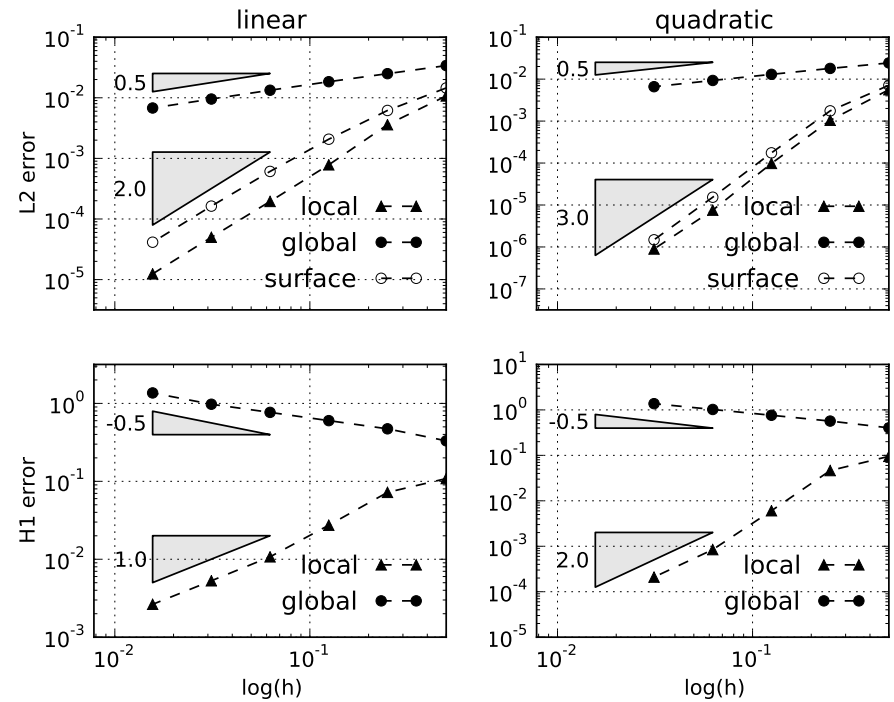

Figure 10: Mesh convergence of WSM for test case II. The figures are analogous to Figure 7 with the addition of the $\boldsymbol{L}^{2}$-norm of the error in the displacement at the traction-free surface.

approximation of the displacement on a $16 \times 16 \times 8$ mesh, for linear and quadratic shape functions, respectively. The displacement fields in the WSM approximations are seen to be continuous everywhere, though they become highly irregular at the dislocation. At further distances from the dislocation, however, the approximations exhibit very good agreement with the exact solution, despite the coarseness of the considered mesh.

Figure 10 examines convergence of the global and local norms of the error under mesh refinement. The results confirm the $O\left(h^{-1 / 2}\right)$ divergence and the $O\left(h^{1 / 2}\right)$ convergence of the global $\boldsymbol{H}^{1}$-norm and global $\boldsymbol{L}^{2}$-norm, respectively, in agreement with the estimates in Corollaries 3 and 5 Furthermore, we observe $O\left(h^{p}\right)$ convergence of the local $\boldsymbol{H}^{1}$-norm in accordance with the estimate in Corollary 7 and $O\left(h^{p+1}\right)$ convergence for the local $\boldsymbol{L}^{2}$-norm. Figure 10 moreover displays the $\boldsymbol{L}^{2}$-norm of the displacement error at the surface, which exhibits an optimal convergence rate of $O\left(h^{p+1}\right)$. It is to be noted that the agreement of the observed convergence rates for the global $\boldsymbol{H}^{1}$-norm and $\boldsymbol{L}^{2}$-norm and the local $\boldsymbol{H}^{1}$-norm with the estimates in Section 4 is non-obvious, as a piecewise constant slip cannot be lifted into $\boldsymbol{H}_{0, \mathcal{D}}^{1}(\Omega \backslash \Gamma)$, and the test case under consideration therefore fails to meet the conditions imposed in Section 4 .

\subsection{Test case III: 3D traction-free rupturing halfspace}

The third test case is in everything equal to the second, except that the dislocation is now extended in vertical direction to span a depth range of $0-0.75$, and the dip slip direction is reversed to form the geodetic equivalent of a rupturing reverse fault; see Figure 11. Figure 12 shows the cross section displacements, and Figure 13 the norms of the displacement-error under mesh refinement. We observe that the global (resp. local) $\boldsymbol{H}^{1}$-norm and $\boldsymbol{L}^{2}$-norm of the error are again proportional to $h^{-1 / 2}$ and $h^{1 / 2}$ (resp. $h^{p}$ and $h^{p+1}$ ), respectively. In addition to the $\boldsymbol{L}^{2}$-norm of the error in 


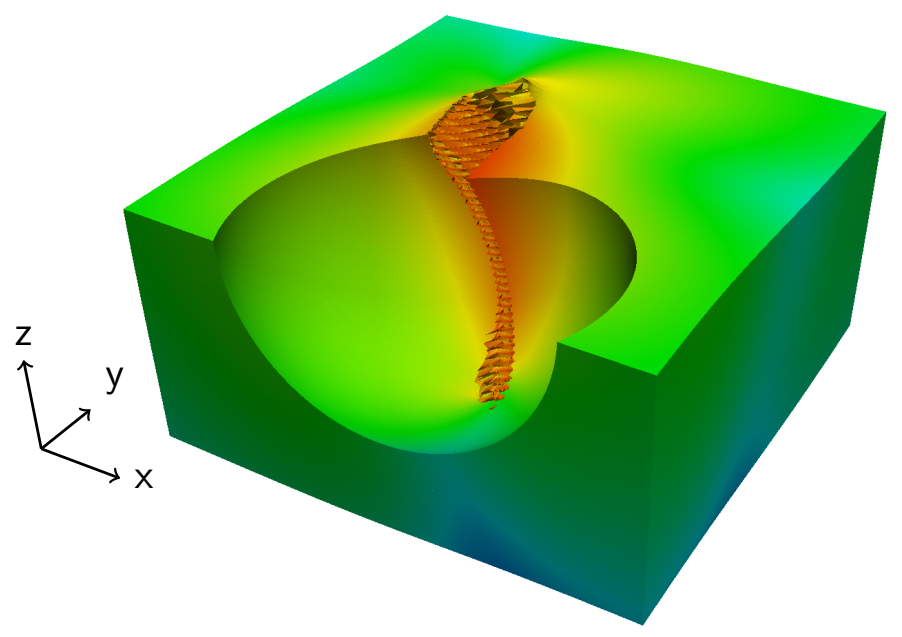

Figure 11: Perspective projection of a WSM computation of test case III, using linear shape functions on a $128 \times 128 \times 64$ mesh. Deformations are amplified by a factor 2 for visualization purposes; colors represent displacement magnitude on a log scale. The orientation aligns roughly with that of Figure 8 A spherical cut-out exposes part of the interior of the domain, revealing a zone of large displacements local to the dislocation.

the displacement field at the traction-free boundary, Figure 13 also presents the local $\boldsymbol{L}^{2}$-norm of the error at the traction-free boundary, i.e., the error on the surface excluding an 0.1-neighborhood of the dislocation. The global $\boldsymbol{L}^{2}$-norm of the surface error converges with an asymptotic rate of $O\left(h^{1 / 2}\right)$. This suboptimal convergence behavior is caused by the fact that in this case the discontinuity reaches the surface. The local $\boldsymbol{L}^{2}$-norm of the error at the surface again display optimal convergence at a rate of $O\left(h^{p+1}\right)$. It is to be noted that the convergence results for the global $\boldsymbol{H}^{1}$-norm and $\boldsymbol{L}^{2}$-norm and the local $\boldsymbol{H}^{1}$-norm agree with the estimates in Section 4 , despite the fact that the analysis in Section 4 is restricted to non-rupturing faults.

\section{Conclusions}

To solve Volterra's dislocation problem by standard finite-elements techniques, the dislocation is required to coincide with element edges. This requirement links the finite-element mesh with the fault geometry, which prohibits the reuse of computational components in situations where multiple geometries have to be considered. In particular, it renders the finite-element method infeasible in nonlinear inversion problems.

To overcome the problems of standard finite-element techniques in nonlinear inversion processes, in this paper we introduced the Weakly-enforced Slip Method (WSM), a new finite-element approximation for Volterra's dislocation problem in which the slip discontinuity is weakly imposed in the right-hand-side load functional. Accordingly, the bilinear form in the formulation and, hence, the stiffness matrix are independent of the fault geometry. The method is summarized by the following 

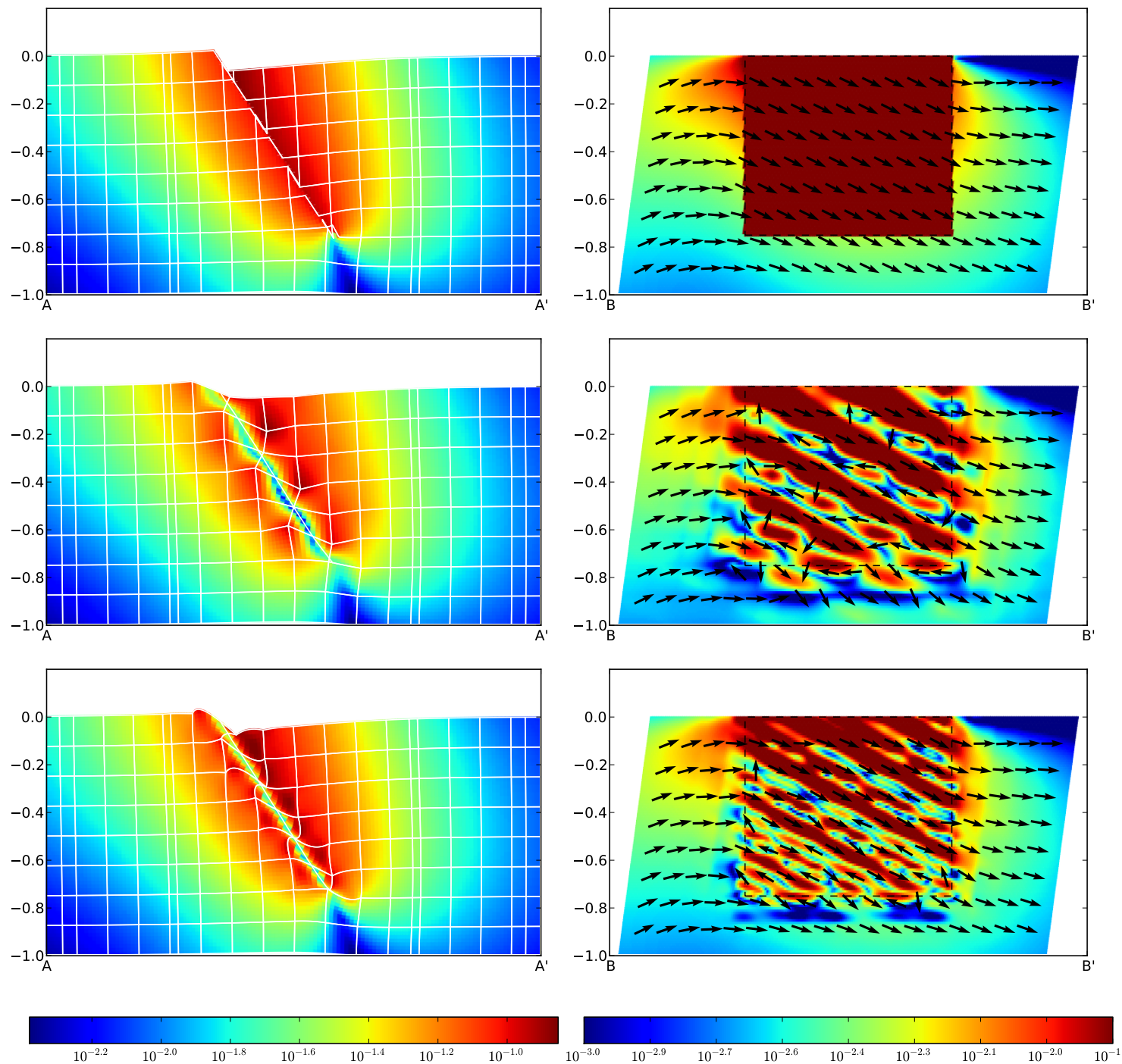

Figure 12: Cross-section views of the displacement field of test case III. The figures are analogous to Figure 7 

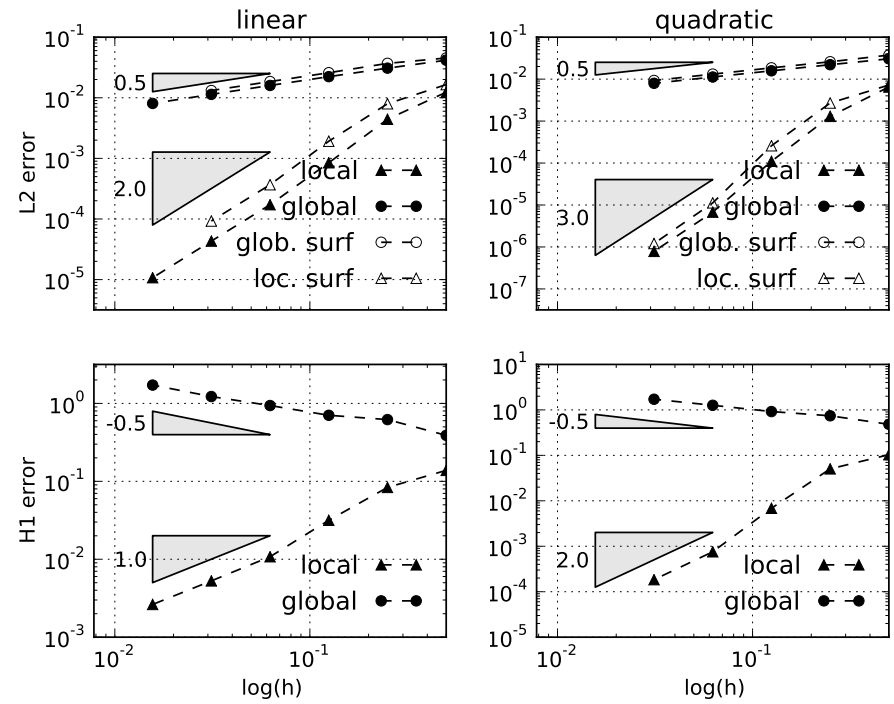

Figure 13: Mesh convergence of WSM applied to test case III. The figures are analogous to Figure 10 except that the $\boldsymbol{L}^{2}$-norm of the error on the surface is now indicated by 'global surface' and additionally a 'local surface' $\boldsymbol{L}^{2}$-norm of the error has been plotted, excluding an 0.1-neighborhood of the dislocation.

weak formulation:

$$
u \in \boldsymbol{V}_{h}: \quad a(u, v)=-\int_{\Gamma} b \cdot\left\{\sigma_{\nu}(v)\right\} \quad \forall v \in \boldsymbol{V}_{h} .
$$

The stiffness matrix depends on properties of the continuous domain only, namely, variations in the elasticity and topology and geometry of the domain, and remains independent of fault geometry. Fault dependence manifests in the right-hand-side load functional only. The load functional is formed by integrating over the fault, which is allowed to cut through elements. The integration along the fault is a non-standard operation in finite-element methods, but we expect that it can be incorporated in most existing finite-element toolkits with minor effort. We further note that no approximations are required regarding fault geometry and slip distribution, unlike lift-based methods, which require a parametrization for both.

We established that as a consequence of the continuous approximation in WSM, the dislocation is not resolved. Accordingly, the WSM approximation displays suboptimal convergence in the $\boldsymbol{L}^{2}$ norm under mesh refinement. In particular, the $\boldsymbol{L}^{2}$-norm of the error decays only as $O\left(h^{1 / 2}\right)$ as the mesh width $h$ tends to 0 , independent of the order of approximation. Furthermore, the $\boldsymbol{H}^{1}$-norm of the error generally diverges as $O\left(h^{-1 / 2}\right)$ as $h \rightarrow 0$, independent of the order of approximation. In addition, we however proved that WSM has outstanding local approximation properties, and that the method generally displays optimal convergence in the $\boldsymbol{H}^{1}$-norm on the domain excluding an arbitrarily small neighborhood of the dislocation. In particular, for any $\varepsilon$-neighborhood $\varkappa^{\varepsilon}$ of the dislocation, the $\|\cdot\|_{1, \Omega \backslash \varkappa^{\varepsilon}}$ norm of the error in the WSM approximation generally converges as $O\left(h^{p}\right)$ as $h \rightarrow 0$, with $p$ the polynomial degree of the finite-element space.

Numerical experiments in 2D and 3D were conducted to verify and scrutinize the approximation properties of WSM. The asymptotic error estimates for the global $\boldsymbol{H}^{1}$-norm, the global $\boldsymbol{L}^{2}$-norm 
and the local $\boldsymbol{H}^{1}$-norm of the error were confirmed in all cases, despite the fact that two of the test cases violate some of the conditions underlying the asymptotic error estimates. In particular, the numerical results indicate that the error estimates extend to rupturing faults, where the dislocation fissures the traction-free surface. The numerical experiments moreover conveyed that the WSM approximation displays optimal local convergence in the $\boldsymbol{L}^{2}$-norm, i.e., the $\|\cdot\|_{\Omega \backslash \varkappa^{\varepsilon} \text {-norm of the }}$ error decays as $O\left(h^{p+1}\right)$ as $h \rightarrow 0$. For the non-rupturing-fault test case, the error in the surface displacement converges optimally in the $\boldsymbol{L}^{2}$-norm at $O\left(h^{p+1}\right)$. For the rupturing-fault test case, the $\boldsymbol{L}^{2}$-norm of the error at the surface converges at $O\left(h^{1 / 2}\right)$, while the local $\boldsymbol{L}^{2}$-norm excluding a small neighborhood of the dislocation again converges optimally at $O\left(h^{p+1}\right)$. Overall, the approximation obtained via WSM is very well behaved, and the method proves remarkably robust.

Given the compelling properties of WSM one might be tempted to seek application in other than our intended field of tectonophysics. Dislocation plasticity comes to mind as one heavily relying on superposition of elastic dislocations. For many problems, however, the location of the fault will be known a-priori, in which case there is no reason to avoid strong imposition. Moreover, often the internal stress is an important quantity to be resolved, which with WSM suffers from inaccuracies in regions close to the fault. In tectonophysics the primary observable is the displacement of the free surface, which WSM is very well capable of resolving.

We believe that WSM can play an important, if specific, role in the application of tectonic fault plane inversions. Being able to precompute the stiffness matrix and a quality preconditioner, for any fault geometry and slip distribution under study, it remains only to integrate over the 2D manifold and solve the system. This makes it feasible to use finite elements in a direct nonlinear inversion. In the hands of geophysicists, this tool will allow all available in-situ knowledge to be made part of the forward model. We hope this will help to improve the accuracy of future co-seismic analyses.

\section{References}

[1] S.C. Brenner and C. Carstensen. Finite element methods. In E. Stein, R. de Borst, and T.J.R. Hughes, editors, Encyclopedia of Computational Mechanics, volume 1: Fundamentals, chapter 4, pages 73-118. John Wiley \& Sons, Ltd., 2004.

[2] S.C. Brenner and L.R. Scott. The Mathematical Theory of Finite Element Methods, volume 15 of Texts in Applied Mathematics. Springer, Berlin, 2nd edition, 2002.

[3] F. Brezzi, T.J.R. Hughes, and E. Süli. Variational approximation of flux in conforming finiteelement methods for elliptic partial differential equations: a model problem. Rend. Mat. Acc. Lincei, 9(12):159-166, 2001.

[4] P.G. Ciarlet. Basic error estimates for elliptic problems. In P.G. Ciarlet and J.-L. Lions, editors, Handbook of Numerical Analysis, volume Vol. II: Finite Element Methods, chapter 2. North-Holland, 1991.

[5] D.A. Di Pietro and A. Ern. Mathematical Aspects of Discontinuous Galerkin Methods. Springer Verlag, 2012.

[6] T. Dupont and R. Scott. Polynomial approximation of functions in Sobolev spaces. Math. Comp., 34, 1980. 
[7] A. Ern and J.-L. Guermond. Theory and Practice of Finite Elements. Number 159 in Applied Mathematical Sciences. Springer-Verlag, New York, 2004.

[8] L.C. Evans. Partial Differential Equations, volume 19 of Graduate Studies in Mathematics. American Mathematical Society, 2009.

[9] R.J. Geller, D.D. Jackson, Y.Y. Kagan, and F. Mulargia. Earthquakes cannot be predicted. Science, 275(5306), 1997.

[10] J.P. Hirth and J. Lothe. Theory of dislocations. Krieger Pub. Co., 4th edition, 1982.

[11] T.J.R. Hughes. The Finite Element Method: Linear Static and Dynamic Finite Element Analysis. Dover, 2000.

[12] R.A. Kerr. New work reinforces megaquake's harsh lessons in geoscience. Science, 332(6032), 2011.

[13] J.-L. Lions and E. Magenes. Non-Homogeous Boundary Value Problems and Applications I. Springer-Verlag, Berlin, 1972.

[14] H.J. Melosh and A. Raefsky. A simple and efficient method for introducing faults into finite element computations. Bulletin of the Seismological Society of America, 71(5), October 1981.

[15] J.A. Nitsche. Über ein variationsprinzip zur lösung Dirichlet-problemen bei verwendung von teilräumen, die keinen randbedingungen unterworfen sind. Abh. Math. Sem. Univ. Hamburg, 36:9-15, 1971.

[16] Y. Okada. Surface deformation due to shear and tensile faults in a half-space. Bulletin of the Seismological Society of America, 75(4), 1985.

[17] Y. Okada. Internal deformation due to shear and tensile faults in a half-space. Bulletin of the Seismological Society of America, 82(2), 1992.

[18] F.-J. Sayas. Weak normal derivatives, normal and tangential traces and tangential differential operators on Lipschitz boundaries. 2009.

[19] A. A. Tronin. Remote sensing and earthquakes: A review. Physics and Chemistry of the Earth, $31,2006$.

[20] E.H. van Brummelen, K.G. van der Zee, V.V. Garg, and S. Prudhomme. Flux evaluation in primal and dual boundary-coupled problems. J. Appl. Mech., 79:010904-8, 2012.

[21] G.J. van Zwieten, R.F. Hanssen, and M.A. Gutiérrez. Overview of a range of solution methods for elastic dislocation problems in geophysics (in press). Journal of Geophysical Research: Solid Earth, (doi:10.1029/2012JB009278), 2013.

[22] O.C. Zienkiewicz and Taylor R.L. The Finite Element Method, volume Volume 2: Solid Mechanics. Butterworth Heinemann, 5th edition, 2000. 